\title{
On an Impulsive Food Web System with Mutual Interference and Distributed Time Delay
}

\author{
Zhen Wang, ${ }^{1}$ Liwei Liu, ${ }^{1}$ Guangwang Su $\mathbb{D}^{1,2}$ and Yuanfu Shao ${ }^{3}$ \\ ${ }^{1}$ School of Science, Nanjing University of Science and Technology, Nanjing 210094, Jiangsu, China \\ ${ }^{2}$ College of Information and Statistics, Guangxi University of Finance and Economics, Nanning, Guangxi 530003, China \\ ${ }^{3}$ College of Science, Guilin University of Technology, Guilin 541004, Guangxi, China
}

Correspondence should be addressed to Guangwang Su; wzailjj@163.com

Received 5 September 2019; Revised 12 November 2019; Accepted 18 November 2019; Published 13 January 2020

Academic Editor: Viktor Avrutin

Copyright ( $\odot 2020$ Zhen Wang et al. This is an open access article distributed under the Creative Commons Attribution License, which permits unrestricted use, distribution, and reproduction in any medium, provided the original work is properly cited.

In this paper, we present a predator-prey system with mutual interference and distributed time delay and study its dynamical behavior. Based on the existence and universality of mutual interference among species, it is necessary to further study an impulsive food web system. By using stability theory, slight perturbation technique, and comparison theorem, we obtain some theoretical results of the system, such as boundedness and permanence. Moreover, numerical experiments are used to verify the theoretical results and to explore the dynamical behavior of the system, which exhibits rich dynamical behavior such as chaotic oscillation, periodic oscillation, symmetry-breaking bifurcations, chaotic crises, and period bifurcation. Finally, we give some practical guidelines for biological systems based on the theoretical results and numerical experiments of the system.

\section{Introduction}

In the past few decades, food web or food chain predatorprey system has been widely studied by many scholars. Many researchers have obtained some classical theoretical knowledge of the predator-prey system and given some practical control strategies, regulation means, and simulation analysis; all these results can be found in these papers [1-4]. With the evolution and development of the ecological system, the relationships of dynamics between predators and prey will continue to be the significant theme of ecology and mathematics, since its existence and importance.

For the predator-prey system, the average consumption rate of a prey by a predator is a hot topic, which is called functional response, and it affects the population relationship between predator and prey. In recent work, for example, these papers [5-7] presented the influence of different functional response functions on the predator-prey system. Moreover, for typical food chain or food web system with an impulsive effect, we can see these papers [8-10]. Specially, some models combined different functional response functions to present the complexity of the system, such as
Sahoo and Poria [11] combined Holling type-I and Holling type-II to study the role of alternative food as a disease controller in a disease-induced predator-prey system. Sahoo and Poria [12] combined general Holling type and Holling type-I to present a diseased prey-predator model, which gave an idea for constructing a realistic food chain model through a proper choice of general Holling parameters. Ghosh et al. [13] discussed the effect of a modified form of Holling typeII response function on additional food for a prey-predator model with prey refuge. In the evolution of biological systems, the state of species changes often occurs at a certain moment. These processes are affected by short-term disturbances; compared with process duration, the state change time always can be neglected $[14,15]$. So it can be assumed that these perturbations are instantaneous; i.e., these perturbations are the form of impulse. Obviously, the impulsive effect is often manifested in the medical mutation rhythm model, biological control model, economic optimization model, frequency-modulated dynamic model, and pharmacokinetics; we can see the related literature in [16-19]; and the papers $[20,21]$ pointed out that impulsive differential equations are a good mathematical tool for describing 
the evolution of these models. In addition, time delay has also been extensively studied in predator-prey systems. In general, two types of time delays have been extensively studied [22]; i.e., continuous/distributed time delay and discrete delay. Some impulsive predator-prey models with distributed time delays are investigated; for example, these papers [23-25] showed some theoretical results and guiding significance of several impulsive systems with distributed time delays. Zhao et al. [26] presented an ecological model with the effect of impulsive and time delay as follows:

$$
\left\{\begin{array}{l}
\frac{\mathrm{d} x_{1}(t)}{\mathrm{d} t}=r x_{1}(t)\left(\frac{k_{0}-x_{1}(t)}{k_{1}-x_{1}(t)}\right)-b_{3} x_{1}^{2}(t)-a_{3} x_{1}(t) x_{2}(t)-\frac{a_{1} x_{1}(t) x_{3}(t)}{b_{1}+x_{1}(t)+c_{1} x_{3}(t)}, \\
\frac{\mathrm{d} x_{2}(t)}{\mathrm{d} t}=d_{1} x_{2}(t) \int_{-\infty}^{t} F(t-s) x_{1}(s) \mathrm{d} s-\frac{a_{2} x_{2}(t) x_{3}(t)}{b_{2}+x_{2}(t)+c_{2} x_{3}(t)}-m_{1} x_{2}(t), \\
\frac{\mathrm{d} x_{3}(t)}{\mathrm{d} t}=\frac{e_{1} a_{1} x_{1}(t) x_{3}(t)}{b_{1}+x_{1}(t)+c_{1} x_{3}(t)}+\frac{e_{2} a_{2} x_{2}(t) x_{3}(t)}{b_{2}+x_{2}(t)+c_{2} x_{3}(t)}-m_{2} x_{3}(t), \\
x_{1}\left(t^{+}\right)-x_{1}(t)=0, \\
x_{2}\left(t^{+}\right)-x_{2}(t)=0, \\
x_{3}\left(t^{+}\right)-x_{3}(t)=p,
\end{array}\right\} t \neq n T,
$$

where $x_{1}(t)$ is the lowest level prey's density, $x_{2}(t)$ is the midlevel predator's density, and $x_{3}(t)$ is the top predator's density at time $t$.

However, in our best knowledge, mutual interference is rarely considered in the predator-prey system. Actually, the phenomenon of mutual interference between predators and prey has always existed, which was studied by Hassell in 1971, and Hassell detailedly introduced the mutual interference constant $m(0<m \leq 1)$. For example, a Volterra system which contains mutual interference is explored by Hassell as follows [27]:

$$
\left\{\begin{array}{l}
x^{\prime}=x g(x)-\psi(x) y^{m}, \\
y^{\prime}=y\left(-d+k \psi(x) y^{m-1}-q(y)\right),
\end{array}\right.
$$

where $\psi(t)$ is a functional response. Compared with the predator-prey system without mutual interference, the predator-prey system with mutual interference contains more dynamic behavior. For example, Wang and Zhu [28] proposed the existence of periodic solutions with mutual interference as follows:

$$
\left\{\begin{array}{l}
x^{\prime}(t)=x(t)\left(a_{1}(t)-b_{1}(t) x(t)\right)-\frac{c_{1}(t) x(t)}{k+x(t)} y^{m}(t), \\
y^{\prime}(t)=y(t)\left(-a_{2}(t)-b_{2}(t) y(t)\right)+\frac{c_{1}(t) x(t)}{k+x(t)} y^{m}(t), \quad m \in(0,1) .
\end{array}\right.
$$

Guo and Chen [29] explored a predator-prey model with mutual interference as follows:

$$
\left\{\begin{array}{l}
x^{\prime}(t)=x(t)\left(a_{1}(t)-b_{1}(t) x(t)\right)-\frac{k_{1}(t) x(t)}{a+b x(t)+c y(t)} y^{m}(t), \\
y^{\prime}(t)=y(t)\left(-a_{2}(t)-b_{2}(t) y(t)\right)+\frac{k_{2}(t) x(t)}{a+b x(t)+c y(t)} y^{m}(t), \quad(0<m \leq 1) .
\end{array}\right.
$$

There are few literatures that present the complex dynamic behavior of mutual interference systems [30-32].

On the one hand, chaos has a serious impact on the management of dynamic population systems. Schaffer and
Kot [33] discussed that chaos is a more complex phenomenon, and controlling chaos is a challenging problem. Sahoo and Poria [34] applied a technique of controlling chaotic predator-prey population dynamics by supplying 
additional food to the top predator, which study is aimed to introduce a new nonchemical chaos control mechanism in a predator-prey system with the applications in fishery management and biological conservation of prey-predator species. The control and management of chaotic population dynamics are one of the main goals of today's ecological mathematical modeling. In our work, we also explore the impact of chaos on complex systems.

In our work, our main purpose is to explore the effect of mutual interference in an impulsive food web with distributed time delays. The food web system described by differential equations is presented as follows:

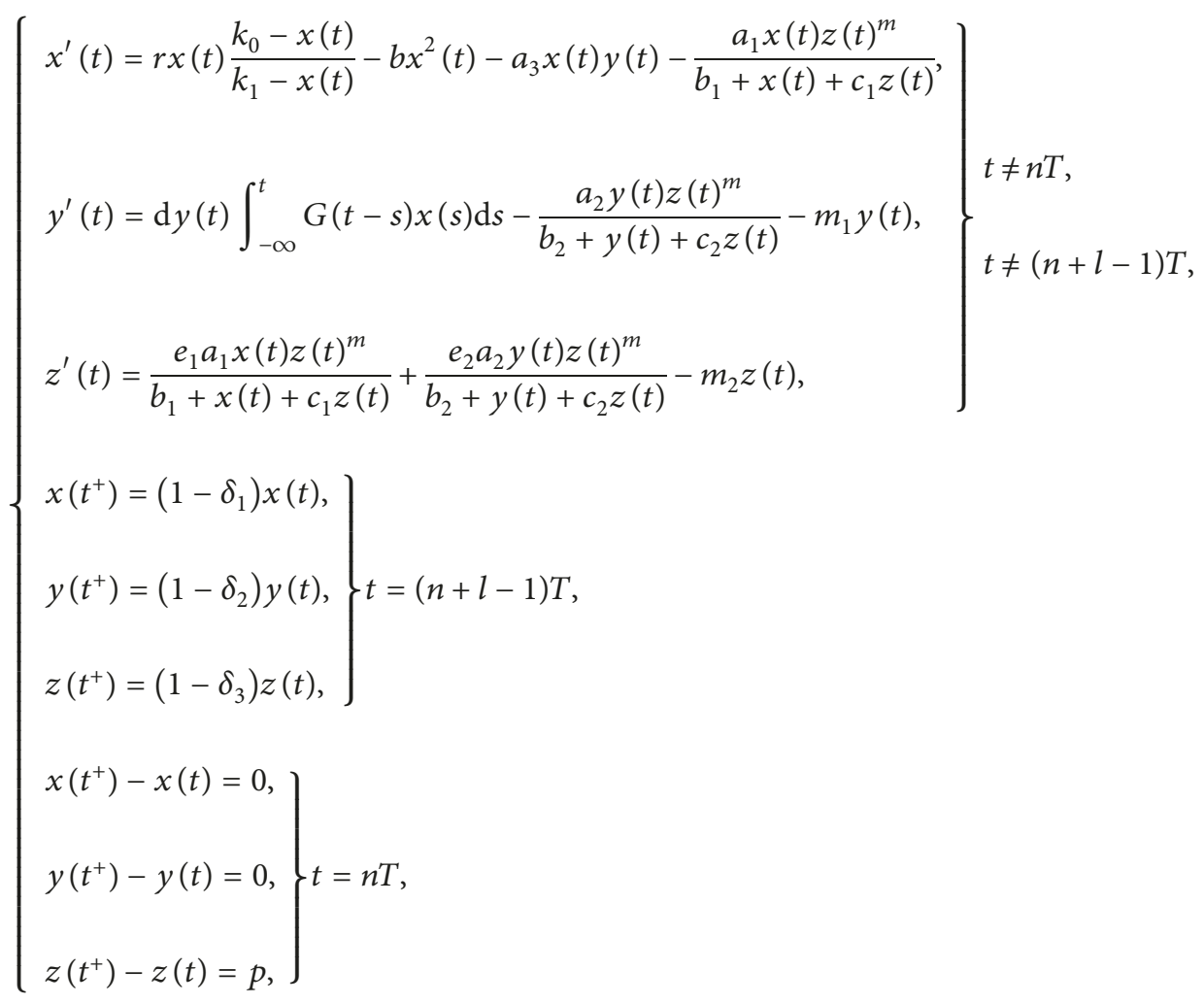

where $x(t), y(t)$, and $z(t)$ represent the densities of bottom prey, medium-level predators, and top level predator at time $t$, respectively. All parameters about the system are positive. Prey's intrinsic increase rate is denoted by $r$. Capture rate of predator to prey was expressed by $a_{i}(i=1,2,3)$. In addition, $e_{i}(i=1,2)$ represents the transformation rate of predator to prey. $m_{1}$ and $m_{2}$ represent the mortality rate of predators $y$ and $z$, respectively. The fraction of available resources was represented by $k_{1} . r k_{0}$ represents the carrying capacity of the prey, where $\left(0 \leq k_{0} / k_{1} \leq 1\right)$. $b$ represents the internal competition rate of prey $x(t) . m$ is the parameter of mutual interference, and its range of values is $[0,1]$. The species are harvested at time $t=(n+l-1) T$, and the fraction of harvest is $\delta_{1}, \delta_{2}$, and $\delta_{3}$, respectively, where their range of values are $[0,1]$. Define $d$ as the product of the conversing rate of prey by predators and average predation rate. Predators $z(t)$ are added to the system at $t=n T$, and the increased amount is $p$. In this mathematical model, the impulsive period is represented by $T$, $0<l<1 ; n$ is the nonnegative integer. In fact, it needs some time for predators to grow by consuming prey, and the time delay occurs in the functional response term, where the function $G(t)=a e^{-a t}$ satisfies $\int_{0}^{+\infty} G(s) \mathrm{d} s=1$ and $a$ is defined as the parameter of distributed time delay.

Now, we can take a chain transformation $q(t)=$ $\int_{-\infty}^{t} G(t-s) x(s) \mathrm{d} s$ to better explore the system (5). Since $\int_{-\infty}^{t} G(t-s) x(s) \mathrm{d} s$ is convergent and

$$
\int_{-\infty}^{t} G(t-s) \mathrm{d} s=\lim _{A \longrightarrow-\infty} \int_{A}^{t} a e^{-a(t-s)} \mathrm{d} s=1,
$$

we can get

$$
\begin{array}{r}
\Delta q(t)=\int_{-\infty}^{t^{+}} G(t-s) x(s) \mathrm{d} s-\int_{-\infty}^{t} G(t-s) x(s) \mathrm{d} s=0, \\
\quad t=n T, t=(n+l-1) T .
\end{array}
$$

Hence, system (5) can be converted to system (8) as follows: 


$$
\left.\left\{\begin{array}{l}
x^{\prime}(t)=r x(t) \frac{k_{0}-x(t)}{k_{1}-x(t)}-b x^{2}(t)-a_{3} x(t) y(t)-\frac{a_{1} x(t) z(t)^{m}}{b_{1}+x(t)+c_{1} z(t)} \\
y^{\prime}(t)=\mathrm{d} y(t) q(t)-\frac{a_{2} y(t) z(t)^{m}}{b_{2}+y(t)+c_{2} z(t)}-m_{1} y(t), \\
z^{\prime}(t)=\frac{e_{1} a_{1} x(t) z(t)^{m}}{b_{1}+x(t)+c_{1} z(t)}+\frac{e_{2} a_{2} y(t) z(t)^{m}}{b_{2}+y(t)+c_{2} z(t)}-m_{2} z(t), \\
q^{\prime}(t)=a x(t)-a q(t), \\
x\left(t^{+}\right)=\left(1-\delta_{1}\right) x(t), \\
y\left(t^{+}\right)=\left(1-\delta_{2}\right) y(t), \\
z\left(t^{+}\right)=\left(1-\delta_{3}\right) z(t), \\
q\left(t^{+}\right)=q(t), \\
z\left(t^{+}\right)=x(t), \\
z\left(t^{+}\right)=q(t), \\
t
\end{array}\right\} t=n+l-1\right) T,
$$

Based on the above discussion, we can explore the dynamic property of system (5) by studying system (8). Hence, system (8) will be mainly considered in this paper.

The remaining sections of our paper are presented as follows. In order to get the theoretical results of this paper, we give some preliminaries in Section 2. In Section 3, the main theoretical results of this article are given. We compare this paper with another paper in Section 4. Numerical experiments are used to explore the mutual interference and the other dynamic property of the system in Section 5. In Section 6, a conclusion and a brief discussion of this article are given.

\section{Preliminaries}

Some preliminaries for proving the theoretical results of systems are given in this section, which is applied to Section 3.

$X(t)=(x(t), y(t), z(t), q(t)): R_{+} \longrightarrow R_{+}^{4}$ is defined as the solution of system (8), which is continuous in interval $((n-1) T,(n+l-1) T) \quad$ and $\quad((n+l-1) T, n T) \quad$ and $R_{+}=[0, \infty), \quad R_{+}^{4}=\{(x(t), y(t), z(t), q(t)) \mid x, y, z, q \geq 0\}$, $(n \in N)$. Define $s$ as the mapping on the right side of system (8). Letting $V: R_{+} \times R_{+}^{4} \longrightarrow R_{+}$, then $V \in V_{0}$, if

(i) For each solution $X(t) \in R_{+}^{4}$, the two limits $\lim _{(t, y)}$ $\longrightarrow\left((n+l-1) T^{+}, X\right) V(t, y)=V\left((n+l-1) T^{+}, X\right)$ and $\lim _{(t, y) \longrightarrow\left(n T^{+}, X\right)} V(t, y)=V\left(n T^{+}, X\right)$ exist, where $V$ is the continuous in interval $((n-1) T,(n+$ $l-1) T] \times R_{+}^{4}$ and $((n+l-1) T, n T] \times R_{+}^{4}, n=1,2 \ldots$

(ii) $V$ is locally Lipschitzian in $X(t)$

Definition 1. If there are two positive constants $m$ and $M$ that make $m \leq x(t), y(t), z(t), q(t) \leq M$, then system (8) is permanent.

Definition 2. Define $D^{+} V(t, X)=\lim _{h \longrightarrow 0^{+}} \sup (1 / h)[V(t+$ $h, X+h f(t, X))-V(t, X)]$ as the upper right derivative of $V(t, X) \quad$ of system (8), where $V \in V_{0}, \quad(t, X) \in$ $((n-1) T,(n+l-1) T] \times R_{+}^{4}$, and $((n+l-1) T, n T] \times R_{+}^{4}$.

Remark 1. $X(t): R_{+} \longrightarrow R_{+}^{4}, X(t)$ is continuous in $((n-1) T,(n+l-1) T) \cup((n+l-1) T, n T), \quad$ and $X\left(t_{0}^{+}\right)=$ $\lim _{t \rightarrow t_{0}^{+}} X(t)$ exists, where $t_{0}=(n+l-l) T^{+}$and $n T^{+}$. The 
solution $X(t)$ is a piecewise continuous function. At the same time, the mapping $f$ with smoothness properties can ensure the global existence and uniqueness of the model (it can see [35]).

Lemma 1 (see [35]). Let $V \in V_{0}, h: R_{+} \times R_{+} \longrightarrow R$, and $h$ is the continuous in interval $((n-1) T,(n+l-1) T]$ and $((n+l-1) T, n T]$. For $g \in R_{+}^{2}, n \in N$, the two limits $\lim _{(t, y) \longrightarrow\left(n T^{+}, g\right)} h(t, y)=h\left(n T^{+}, g\right)$ and $\lim _{(t, y) \longrightarrow\left((n+l-1) T^{+}, g\right)}$ $h(t, y)=h\left((n+l-1) T^{+}, g\right)$ exist. Suppose that $\psi_{n}^{1}, \psi_{n}^{2}: R_{+}$ $\longrightarrow R_{+}$, which are nondecreasing function for all $n \in N$. And assume that, when $t \neq n T,(n+l-1) T$,

$$
D^{+} V(t, X) \leq h(t, V(t, X)) \text {. }
$$

When $t=(n+l-1) T$,

$$
V\left(t, X\left(t^{+}\right)\right) \leq \psi_{n}^{1}(V(t, X)),
$$

and when $t=n T$,

$$
V\left(t, X\left(t^{+}\right)\right) \leq \psi_{n}^{2}(V(t, X)) .
$$

Suppose that $r(t)$ be the maximal solution of the following impulsive differential:

$$
\left\{\begin{array}{l}
g^{\prime}(t)=h(t, g(t)), \quad t \neq n T, t \neq(n+l-1) T, \\
g\left(t^{+}\right)=\psi_{n}^{1}(g(t)), \quad t=(n+l-1) T, \\
g\left(t^{+}\right)=\psi_{n}^{2}(g(t)), \quad t=n T, \\
g\left(0^{+}\right)=g_{0} \geq 0,
\end{array}\right.
$$

which exists on $[0, \infty)$. Then, the inequality $V\left(0^{+}, X_{0}\right) \leq g_{0}$ holds, which shows that the inequality $V(t, X(t)) \leq r(t)$ (all $t \geq 0$ ) holds.

Now, we study the following system, which is the subsystem of system (8):

$$
\begin{cases}z^{\prime}(t)=-m_{2} z(t), & t \neq n T, t \neq(n+l-1) T, \\ z\left(t^{+}\right)=\left(1-\delta_{3}\right) z(t), & t=(n+l-1) T, \\ z\left(t^{+}\right)=z(t)+p, & t=n T, \\ z\left(0^{+}\right)=z_{0} . & \end{cases}
$$

After some algebraic calculations, we can get positive periodic solution of this subsystem as follows:

$$
\begin{gathered}
z^{*}(t)= \begin{cases}\frac{p \exp \left(-m_{2}(t-(n-1) T)\right)}{1-\left(1-\delta_{3}\right) \exp \left(-m_{2} T\right)}, & (n-1) T<t \leq(n+l-1) T, \\
\frac{p\left(1-\delta_{3}\right) \exp \left(-m_{2}(t-(n-1) T)\right)}{1-\left(1-\delta_{3}\right) \exp \left(-m_{2} T\right)}, & (n+l-1) T<t \leq n T,\end{cases} \\
\left(n \in N, z^{*}\left(0^{+}\right)=z^{*}\left(n T^{+}\right)=\frac{p}{1-\left(1-\delta_{3}\right) \exp \left(-m_{2} T\right)},\right. \\
\left.z^{*}\left((n+l-1) T^{+}\right)=z^{*}\left(l T^{+}\right)=\frac{p\left(1-\delta_{3}\right) \exp \left(-m_{2} l T\right)}{1-\left(1-\delta_{3}\right) \exp \left(-m_{2} T\right)}\right),
\end{gathered}
$$

which is a positive periodic solution of system (13). Since

$$
z(t)=\left\{\begin{array}{l}
\left(1-\delta_{3}\right)^{n-1}\left(z\left(0^{+}\right)-\frac{p}{1-\left(1-\delta_{3}\right) \exp \left(-m_{2} T\right)}\right) \exp \left(-m_{2} t\right)+z^{*}(t), \\
(n-1) T<t \leq(n+l-1) T, \\
\left(1-\delta_{3}\right)^{n}\left(z\left(0^{+}\right)-\frac{p}{1-\left(1-\delta_{3}\right) \exp \left(-m_{2} T\right)}\right) \exp \left(-m_{2} t\right)+z^{*}(t), \\
(n+l-1) T<t \leq n T,
\end{array}\right.
$$


is the solution of system (13) with initial value $z\left(0^{+}\right) \geq 0$, we can get following lemma.

Lemma 2 (see [35]). Suppose $z^{*}(t)$ is a positive periodic solution of system (13), for each solution $z(t)$ with $z\left(0^{+}\right) \geq 0$, it follows that $\left|z(t)-z^{*}(t)\right| \longrightarrow 0$ as $t \longrightarrow \infty$.

Lemma 3 (see [36]). Suppose that function $V(t) \in P C^{1}$ $\left(R_{+}, R\right)$ satisfies the following equation:

$$
\left\{\begin{array}{l}
\frac{\mathrm{d} V}{\mathrm{~d} t} \leq w(t) V(t)+h(t), \quad t \neq t_{k}, t>0, \\
V\left(t_{k}^{+}\right) \leq j_{k} V\left(t_{k}\right)+l_{k}, \quad t=t_{k}>0, \\
g\left(0^{+}\right) \leq g_{0},
\end{array}\right.
$$

where $w, h \in C\left(R_{+}, R\right), \quad j_{k} \geq 0$, and $l_{k}$ are constants $(k=1,2, \ldots)$. For $t>0$, we have

$$
\begin{aligned}
V(t) \leq & V_{0}\left(\prod_{t_{0}<t_{k}<t} j_{k}\right) \exp \left(\int_{t_{0}}^{t} w(s) \mathrm{d} s\right)+\int_{t_{0}}^{t}\left(\prod_{t_{0}<t_{k}<t} j_{k}\right) \\
& \exp \left(\int_{s}^{t} w(\gamma) \mathrm{d} \gamma\right) h(s) \mathrm{d} s+\sum_{t_{0}<t_{k}<t}\left(\prod_{t_{k}<t_{j}<t} j_{j}\right) \\
& \exp \left(\int_{t_{k}}^{t} w(s) \mathrm{d} s\right) l_{k} .
\end{aligned}
$$

Next, we explore boundedness, globally asymptotically stable, and permanence of system (8) in next sections.

\section{Main Theoretical Results}

\subsection{Boundedness of System (8)}

Theorem 1. Let $(x(t), y(t), z(t), q(t))$ be the solution of system (8), and it always can find a positive constant $M$, which makes $x(t) \leq M, y(t) \leq M, z(t) \leq M$, and $q(t) \leq M$ hold.

Proof. For the solution $X(t)=(x(t), y(t), z(t), q(t))$ of model (8) with initial value $\left(x_{0}, y_{0}, z_{0}, q_{0}\right)$, let $V(t, X(t))=e_{1} x(t)+e_{2} y(t)+z(t)+q(t)$, where $V \in V_{0}$. Since

$$
\left\{\begin{array}{l}
\frac{\mathrm{d} x(t)}{\mathrm{d} t} \leq r x(t) \frac{k_{0}-x(t)}{k_{1}-x(t)}-b x^{2}(t) \leq x(t)(r-b x(t)), \\
q(t)=\int_{-\infty}^{t} F(t-s) x(s) \mathrm{d} s
\end{array}\right.
$$

we can obtain $x(t), q(t) \leq(r / b)$. Choose $0<\lambda<\min$ $\left\{m_{1}-(r d / b), m_{2}, a\right\}$, we can get the following inequality relations:

$$
\begin{aligned}
D^{+} V(t)+\lambda V(t) \leq & -e_{1} b x^{2}(t)+\lambda e_{1} x(t)+a x(t)+e_{1} r x(t) \\
& +\left(\lambda e_{2}+\frac{r e_{2} d}{b}-e_{2} m_{1}\right) y(t)+\left(\lambda-m_{2}\right) z(t) \\
& +(\lambda-a) q(t) \leq M_{0}, \quad t \neq(n+l-1) T, t \neq n T,
\end{aligned}
$$

where

$$
M_{0}=\frac{\left(\lambda e_{1}+a+e_{1} r\right)^{2}}{4 e_{1} b} .
$$

In addition, clearly,

$$
\begin{cases}V\left((n+l-1) T^{+}\right) \leq V((n+l-1) T), & t=(n+l-1) T ; \\ V\left(n T^{+}\right) \leq V(n T)+p, & t=n T .\end{cases}
$$

By using Lemma 3, we can obtain

$$
\begin{aligned}
D^{+} V(t)+\lambda V(t) \leq & -e_{1} b x^{2}(t)+\lambda e_{1} x(t)+a x(t)+e_{1} r x(t) \\
& +\left(\lambda e_{2}+\frac{r e_{2} d}{b}-e_{2} m_{1}\right) y(t)+\left(\lambda-m_{2}\right) z(t) \\
& +(\lambda-a) q(t) \leq M_{0} .
\end{aligned}
$$

To sum up the above discussion, for sufficiently large $t$, $V(t, X(t))$ is bounded; i.e., $x(t) \leq M, y(t) \leq M, z(t) \leq M$, and $q(t) \leq M$ hold for sufficiently large $t$

\subsection{Globally Asymptotically Stable Solution $\left(0,0, z^{*}(t), 0\right)$}

Theorem 2. Let $\left(0,0, z^{*}(t), 0\right)$ be the eradication solution of prey and intermediate predator of system (8), which is globally asymptotically stable if and only if the following two inequalities hold:

$$
\begin{aligned}
& \frac{r k_{0} T}{k_{1}}+\frac{a_{1}}{c_{1} m m_{2}}\left(A_{1}+A_{2}\right)<\ln \frac{1}{1-\delta_{1}}, \\
& -m_{1} T+\frac{a_{2}}{c_{2} m m_{2}}\left(B_{1}+B_{2}\right)<\ln \frac{1}{1-\delta_{2}},
\end{aligned}
$$

where 


$$
\begin{aligned}
& A=\left(1-\left(1-\delta_{3}\right) \exp \left(-m_{2} T\right)\right)^{m}, \\
& A_{1}=\ln \left(1-\frac{c_{1} p^{m}\left(1-\exp \left(-m_{2} m l T\right)\right)}{b_{1} A+c_{1} p^{m}}\right), \\
& A_{2}=\ln \left(1-\frac{c_{1} p^{m}\left(1-\delta_{3}\right)^{m}\left(\exp \left(-\operatorname{lm}{ }_{2} m T\right)-\exp \left(-m_{2} m T\right)\right)}{b_{1} A+c_{1} p^{m}\left(1-\delta_{3}\right)^{m} \exp \left(-\operatorname{lm} m_{2} m T\right)}\right) \\
& B_{1}=\ln \left(1-\frac{c_{2} p^{m}\left(1-\exp \left(-\operatorname{lm} m_{2} m T\right)\right)}{b_{2} A+c_{2} p^{m}}\right), \\
& B_{2}=\ln \left(1-\frac{c_{2} p^{m}\left(1-\delta_{3}\right)^{m}\left(\exp \left(-l m_{2} m T\right)-\exp \left(-m_{2} m T\right)\right)}{b_{2} A+c_{2} p^{m}\left(1-\delta_{3}\right)^{m} \exp \left(-\operatorname{lm} m_{2} m T\right)}\right) .
\end{aligned}
$$

Proof. Let $X(t)=(x(t), y(t), z(t), q(t))$ be the solution of system (8), by taking the technique of small amplitude perturbations for the solution, and we can derive the local stability of periodic solutions $\left(0,0, z^{*}(t), 0\right)$. Let $x(t)=u_{1}$ $(t), y(t)=u_{2}(t), z(t)=z^{*}(t)+u_{3}(t)$, and $q(t)=u_{4}(t)$, where $u_{i}(t)(i=1,2,3,4)$ is a small perturbation. When $t \neq n T,(n+l-1) T$, by applying Taylor series expansion and neglecting higher order terms, system (8) can be read as follows:

$$
\left\{\begin{array}{l}
u_{1}^{\prime}(t)=\left(\frac{r k_{0}}{k_{1}}-\frac{a_{1} z^{*}(t)^{m}}{b_{1}+c_{1} z^{*}(t)}\right) u_{1}(t), \\
u_{2}^{\prime}(t)=\left(-\frac{a_{2} z^{*}(t)^{m}}{b_{2}+c_{2} z^{*}(t)}-m_{1}\right) u_{2}(t), \\
u_{3}^{\prime}(t)=\frac{e_{1} a_{1} z^{*}(t)^{m} u_{1}(t)}{b_{1}+c_{1} z^{*}(t)}+\frac{e_{2} a_{2} z^{*}(t)^{m} u_{2}(t)}{b_{2}+c_{2} z^{*}(t)}-m_{2} u_{3}(t), \\
u_{4}^{\prime}(t)=a u_{1}(t)-a u_{4}(t), \\
u_{1}\left(t^{+}\right)=\left(1-\delta_{1}\right) u_{1}(t), \\
u_{2}\left(t^{+}\right)=\left(1-\delta_{2}\right) u_{2}(t), \\
u_{3}\left(t^{+}\right)=\left(1-\delta_{3}\right) u_{3}(t), \\
u_{4}\left(t^{+}\right)=u_{4}(t), \\
u_{1}\left(t^{+}\right)=u_{1}(t),
\end{array}\right\} t=(n+l-1) T,
$$


so we can get

$$
\left(\begin{array}{l}
u_{1}(t) \\
u_{2}(t) \\
u_{3}(t) \\
u_{4}(t)
\end{array}\right)=\Phi(t)\left(\begin{array}{l}
u_{1}(0) \\
u_{2}(0) \\
u_{3}(0) \\
u_{4}(0)
\end{array}\right), \quad 0 \leq t<T
$$

where $\Phi(t)$ is the fundamental matrix of (26). At the same time, $\Phi(t)$ satisfies

$$
\frac{\mathrm{d} \Phi(t)}{\mathrm{d} t}=\left(\begin{array}{cccc}
\frac{r k_{0}}{k_{1}}-\frac{a_{1} z^{*}(t)^{m}}{b_{1}+c_{1} z^{*}(t)} & 0 & 0 & 0 \\
0 & -\frac{a_{2} z^{*}(t)^{m}}{b_{2}+c_{2} z^{*}(t)}-m_{1} & 0 & 0 \\
\frac{e_{1} a_{1} z^{*}(t)^{m}}{b_{1}+c_{1} z^{*}(t)} & \frac{e_{2} a_{2} z^{*}(t)^{m}}{b_{2}+c_{2} z^{*}(t)} & -m_{2} & 0 \\
a & 0 & 0 & -a
\end{array}\right) \Phi(t) .
$$

Let $t=0$, then $\Phi(0)=I$ is a identity matrix, so we can get the linearized structure of system (8) as follows:

$$
\begin{aligned}
& \left(\begin{array}{c}
u_{1}\left((n+l-1) T^{+}\right) \\
u_{2}\left((n+l-1) T^{+}\right) \\
u_{3}\left((n+l-1) T^{+}\right) \\
u_{4}\left((n+l-1) T^{+}\right)
\end{array}\right)=\left(\begin{array}{cccc}
1-\delta_{1} & 0 & 0 & 0 \\
0 & 1-\delta_{2} & 0 & 0 \\
0 & 0 & 1-\delta_{3} & 0 \\
0 & 0 & 0 & 1
\end{array}\right) \\
& \left(\begin{array}{c}
u_{1}((n+l-1) T) \\
u_{2}((n+l-1) T) \\
u_{3}((n+l-1) T) \\
u_{4}((n+l-1) T)
\end{array}\right) \\
& \left(\begin{array}{l}
u_{1}\left(n T^{+}\right) \\
u_{2}\left(n T^{+}\right) \\
u_{3}\left(n T^{+}\right) \\
u_{4}\left(n T^{+}\right)
\end{array}\right)=\left(\begin{array}{llll}
1 & 0 & 0 & 0 \\
0 & 1 & 0 & 0 \\
0 & 0 & 1 & 0 \\
0 & 0 & 0 & 1
\end{array}\right)\left(\begin{array}{l}
u_{1}(n T) \\
u_{2}(n T) \\
u_{3}(n T) \\
u_{4}(n T)
\end{array}\right) .
\end{aligned}
$$

By using the eigenvalues of following matrix $M$, we can derive the stability of the solutions $\left(0,0, z^{*}(t), 0\right)$ :

$$
M=\left(\begin{array}{cccc}
1-\delta_{1} & 0 & 0 & 0 \\
0 & 1-\delta_{2} & 0 & 0 \\
0 & 0 & 1-\delta_{3} & 0 \\
0 & 0 & 0 & 1
\end{array}\right)\left(\begin{array}{llll}
1 & 0 & 0 & 0 \\
0 & 1 & 0 & 0 \\
0 & 0 & 1 & 0 \\
0 & 0 & 0 & 1
\end{array}\right) \Phi(T) .
$$

If $\lambda_{i}<1 \quad(i=1,2,3,4)$, then the periodic solution $\left(0,0, z^{*}(t), 0\right)$ is locally stable, and their expressions are as follows:

$$
\begin{aligned}
& \lambda_{1}=\left(1-\delta_{1}\right) \exp \left(\int_{0}^{T}\left(\frac{r k_{0}}{k_{1}}-\frac{a_{1} z^{*}(t)^{m}}{b_{1}+c_{1} z^{*}(t)}\right) \mathrm{d} t\right), \\
& \lambda_{2}=\left(1-\delta_{2}\right) \exp \left(\int_{0}^{T}\left(-\frac{a_{2} z^{*}(t)^{m}}{b_{2}+c_{2} z^{*}(t)}-m_{1}\right) \mathrm{d} t\right), \\
& \lambda_{3}=\left(1-\delta_{3}\right) \exp \left(-m_{2} T\right) \\
& \lambda_{4}=\exp (-a T)
\end{aligned}
$$

The analysis is similar to literature [35], i.e., by using Floquet theory, if $\left|\lambda_{i}\right|<1(i=1,2,3,4)$, then $\left(0,0, z^{*}(t), 0\right)$ is locally asymptotically stable. So we need to calculate $\left|\lambda_{i}\right|<1(i=1,2)$; herein, $\lambda_{3}$ and $\lambda_{4}$ are already less than one. Therefore, we have

$$
\begin{aligned}
& \lambda_{1}<1 \Longleftrightarrow T<\frac{k_{1}}{r k_{0}}\left(\ln \frac{1}{1-\delta_{1}}-\frac{a_{1}}{c_{1} m m_{2}}\left(A_{1}+A_{2}\right)\right), \\
& \lambda_{2}<1 \Longleftrightarrow T>\frac{1}{-m_{1}}\left(\ln \frac{1}{1-\delta_{2}}-\frac{a_{2}}{c_{2} m m_{2}}\left(B_{1}+B_{2}\right)\right),
\end{aligned}
$$

where

$$
\begin{aligned}
\int_{0}^{T} z^{*}(t)^{m} \mathrm{~d} t= & \int_{(n-1) T}^{(n+l-1) T}\left(\frac{p \exp \left(-m_{2}(t-(n-1) T)\right)}{1-\left(1-\delta_{3}\right) \exp \left(-m_{2} T\right)}\right)^{m} \mathrm{~d} t \\
& +\int_{(n+l-1) T}^{n T}\left(\frac{p\left(1-\delta_{3}\right) \exp \left(-m_{2}(t-(n-1) T)\right)}{1-\left(1-\delta_{3}\right) \exp \left(-m_{2} T\right)}\right)^{m} \mathrm{~d} t .
\end{aligned}
$$

Inequality (32) holds if and only if

$$
\left\{\begin{array}{l}
\frac{r k_{0} T}{k_{1}}+\frac{a_{1}}{c_{1} m m_{2}}\left(A_{1}+A_{2}\right)<\ln \frac{1}{1-\delta_{1}}, \\
-m_{1} T+\frac{a_{2}}{c_{2} m m_{2}}\left(B_{1}+B_{2}\right)<\ln \frac{1}{1-\delta_{2}} .
\end{array}\right.
$$

Hence, when the inequality (34) is established, the system (8) is locally asymptotically stable.

After the above discussion, we can obtain the conditions of local asymptotic stability of periodic solutions $\left(0,0, z^{*}(t), 0\right)$. Now, we explore global attraction of the periodic solutions; for $\varepsilon>0$, we have

$$
\begin{aligned}
& \xi_{1} \triangleq\left(1-\delta_{1}\right) \exp \left(\int_{(n+l-1) T}^{(n+l) T}\left(\frac{r k_{0}}{k_{1}}-\frac{a_{1}\left(z^{*}(t)-\varepsilon\right)^{m}}{b_{1}+c_{1}\left(z^{*}(t)-\varepsilon\right)}\right) \mathrm{d} t\right)<1, \\
& \xi_{2} \triangleq\left(1-\delta_{2}\right) \exp \left(\int_{0}^{T}\left(\mathrm{~d} M-m_{1}-\frac{a_{2}\left(z^{*}(t)-\varepsilon\right)^{m}}{b_{2}+c_{2}\left(z^{*}(t)-\varepsilon\right)}\right) \mathrm{d} t\right)<1 .
\end{aligned}
$$

Noticing that $(\mathrm{d} z(t) / \mathrm{d} t) \geq-m_{2} z(t)$, according to Lemma 2 and comparison theorem of the impulsive equation, for all sufficiently large $t$, we can obtain

$$
z(t)>z^{*}(t)-\varepsilon \text {. }
$$

For all $t>0$, assuming that inequality (36) is established, so we consider the system (8) and inequality (36), we can get 


$$
\begin{cases}\frac{\mathrm{d} x(t)}{\mathrm{d} t} \leq\left(\frac{r k_{0}}{k_{1}}-\frac{a_{1}\left(z^{*}(t)-\varepsilon\right)^{m}}{b_{1}+c_{1}\left(z^{*}(t)-\varepsilon\right)}\right) x(t), & t \neq(n+l-1) T, \\ x\left(t^{+}\right)=\left(1-\delta_{1}\right) x_{1}(t), & t=(n+l-1) T,\end{cases}
$$

integrating (37) on $((n+l-1) T,(n-1) T]$, and from (8), we have

$$
\begin{aligned}
& x((n+l) T) \leq x\left((n+l-1) T^{+}\right) \\
& \cdot \exp \left(\int_{(n+l-1) T}^{(n+l) T}\left(\frac{r k_{0}}{k_{1}}-\frac{a_{1}\left(z^{*}(t)-\varepsilon\right)^{m}}{b_{1}+c_{1}\left(z^{*}(t)-\varepsilon\right)}\right) \mathrm{d} t\right), \\
& =x((n+l-1) T)\left(1-\delta_{1}\right) \exp \int_{(n+l-1) T}^{(n+l) T} \\
& \cdot\left(\left(\frac{r k_{0}}{k_{1}}-\frac{a_{1}\left(z^{*}(t)-\varepsilon\right)^{m}}{b_{1}+c_{1}\left(z^{*}(t)-\varepsilon\right)}\right) \mathrm{d} t\right), \\
& =x((n+l-1) T) \xi_{1} .
\end{aligned}
$$

After some algebraic calculations of (38), which produces $x((n+l) T) \leq x(l T) \xi_{1}^{n}$, when $n \longrightarrow \infty$, it follows that $x((n+l) T) \longrightarrow 0$. When $(n+l-1) T<t \leq(n+l) T$, we get $0<x(t)<x((n+l-1) T)\left(1-\delta_{1}\right) \exp (r T)$, so $x(t) \longrightarrow 0$ as $n \longrightarrow \infty$. In addition, if $x(t) \longrightarrow 0$, which produces $q(t) \longrightarrow 0$. Then, for $y(t)$, we have

$$
\begin{cases}\frac{\mathrm{d} y(t)}{\mathrm{d} t} \leq\left(\mathrm{d} M-m_{1}-\frac{a_{2}\left(z^{*}(t)-\varepsilon\right)^{m}}{b_{2}+c_{2}\left(z^{*}(t)-\varepsilon\right)}\right) y(t), & t \neq(n+l-1) T, \\ y\left(t^{+}\right)=\left(1-\delta_{2}\right) y_{1}(t), & t=(n+l-1) T .\end{cases}
$$

The analysis is similar to $x(t)$, and it can also obtain $y(t) \longrightarrow 0$ as $n \longrightarrow \infty$.
Next, our goal is to explore $z(t)$ to $z^{*}(t)(t \longrightarrow \infty)$. Let $0<\varepsilon<\left(r_{3} / \gamma\right)\left(\gamma=\left(e_{1} a_{1} / b_{1}\right)+\left(e_{2} a_{2} / b_{2}\right)\right)$, we can find a $T^{\prime}>0$, which produces $0<x(t), y(t), q(t)<\varepsilon$ as $t>T^{\prime}$. Actually, assume that $0<x(t), y(t), q(t)<\varepsilon$ for all $t \geq 0$. Then, we have the following inequality by system (8):

$$
\begin{aligned}
-m_{2} z(t) & \leq \frac{\mathrm{d} z(t)}{\mathrm{d} t} \leq-m_{2} z(t)+\gamma \mathcal{E} z(t)^{m}, \\
\gamma & =\frac{e_{1} a_{1}}{b_{1}}+\frac{e_{2} a_{2}}{b_{2}} .
\end{aligned}
$$

Because $0<m \leq 1$, the inequality (40) can be changed to $-m_{2} z(t) \leq(\mathrm{d} z(t) / \mathrm{d} t) \leq\left(-m_{2}+\gamma \varepsilon\right) z(t)$. We now consider comparative systems (41) and (42):

$$
\begin{aligned}
& \left\{\begin{array}{l}
\frac{\mathrm{d} v_{1}(t)}{\mathrm{d} t}=-m_{2} v_{1}(t), \quad t \neq n T, t \neq(n+l-1) T \\
v_{1}\left(t^{+}\right)=\left(1-\delta_{3}\right) v_{1}(t), \quad t=(n+l-1) T \\
v_{1}\left(t^{+}\right)=v_{1}(t)+p, \quad t=n T, \\
v_{1}\left(0^{+}\right)=z\left(0^{+}\right), \\
\left\{\begin{array}{l}
\frac{\mathrm{d} v_{2}(t)}{\mathrm{d} t}=\left(-m_{2}+\gamma \varepsilon\right) v_{2}(t), \quad t \neq n T, t \neq(n+l-1) T \\
v_{2}\left(t^{+}\right)=\left(1-\delta_{3}\right) v_{2}(t), \quad t=(n+l-1) T \\
v_{2}\left(t^{+}\right)=v_{2}(t)+p, \\
v_{2}\left(0^{+}\right)=z\left(0^{+}\right),
\end{array} \quad t=n T\right.
\end{array}\right.
\end{aligned}
$$

let $v_{1}(t)$ and $v_{2}(t)$ be any solution of system (41) and (42), respectively, where

$$
v_{2}^{*}(t)= \begin{cases}\frac{p \exp \left(\left(-m_{2}+\gamma \varepsilon\right)(t-(n-1) T)\right)}{1-\left(1-\delta_{3}\right) \exp \left(\left(-m_{2}+\gamma \varepsilon\right) T\right)}, & (n-1) T<t \leq(n+l-1) T, \\ \frac{p\left(1-\delta_{3}\right) \exp \left(\left(-m_{2}+\gamma \varepsilon\right)(t-(n-1) T)\right)}{1-\left(1-\delta_{3}\right) \exp \left(\left(-m_{2}+\gamma \varepsilon\right) T\right)}, & (n+l-1) T<t \leq n T .\end{cases}
$$

By using Lemmas 1 and 2, which can derive $v_{1}(t) \leq z(t) \leq v_{2}(t)$. Furthermore, when $t \longrightarrow \infty$, $v_{1}(t) \longrightarrow z^{*}(t)$ and $v_{2}(t) \longrightarrow v_{2}^{*}(t)$. Then, $\exists T_{1}>0$ for any $\varepsilon_{1}>0$, and it yields the following inequalities:

$$
z^{*}(t)-\varepsilon_{1}<z(t)<z^{*}(t)+\varepsilon, \quad t \geq T_{1} .
$$

Obviously, $v_{2}^{*}(t) \longrightarrow z^{*}(t)$ as $\varepsilon \longrightarrow 0$. Therefore, for $t$ large enough, it yields $z^{*}(t)-\varepsilon_{1}<z(t)<z^{*}(t)+\varepsilon_{1}$, which implies $z(t) \longrightarrow z^{*}(t)$ as $t \longrightarrow \infty$.

\subsection{Permanence of the System}

Theorem 3. If the following two inequalities hold, then system (8) is permanent:

$$
\begin{aligned}
& \frac{r k_{0} T}{k_{1}}+\frac{a_{1}}{c_{1} m m_{2}}\left(A_{1}+A_{2}\right)>\ln \frac{1}{1-\delta_{1}}, \\
& -m_{1} T+\frac{a_{2}}{c_{2} m m_{2}}\left(B_{1}+B_{2}\right)>\ln \frac{1}{1-\delta_{2}},
\end{aligned}
$$

where $A, A_{1}, A_{2}, B_{1}$, and $B_{2}$ are given in Theorem 2 . 
Proof. From Theorem 1, we can know that there is a constant $M$ which makes $x(t), y(t), z(t), q(t)<M$ for $t \geq 0$. Since $z^{\prime}(t) \geq-m_{2} z(t)$, we give the following differential equation (46):

$$
\begin{cases}v_{3}^{\prime}(t)=-m_{2} v_{3}(t), & t \neq n T, t \neq(n+l-1) T, \\ v_{3}\left(t^{+}\right)=\left(1-\delta_{3}\right) v_{3}(t), & t=(n+l-1) T \\ v_{3}\left(t^{+}\right)=v_{3}(t)+p, & t=n T, \\ v_{3}\left(0^{+}\right)=z\left(0^{+}\right)>0 . & \end{cases}
$$

By Lemmas 1 and 2, for sufficiently large $t \geq 0$, we can get $z(t) \geq v_{3}(t)>z^{*}(t)-\varepsilon$ and $z(t) \geq\left(p\left(1-\delta_{3}\right) \exp \left(-m_{2} T\right) / 1\right.$ $\left.-\left(1-\delta_{3}\right) \exp \left(-m_{2} T\right)\right)-\varepsilon_{2}=m_{3}\left(\varepsilon_{2}>0\right)$. So our next goal is to find positive $m_{1}$ and $m_{2}$ such that $x(t) \geq m_{1}, y(t) \geq m_{2}$, and $q(t) \geq m_{1}$ for $t$ large enough. Now we are exploring how to find $m_{1}$.

The following two steps prove the existence of such a $m_{1}$. Firstly, let $0<\overline{m_{1}}<\left(\left(b_{1} m_{2} / e_{1} a_{1} M^{m-1}\right),\left(b_{2} m_{2} / e_{2} a_{2} M^{m-1}\right)\right)$ and sufficiently small $\varepsilon_{1}>0$, then we have

$$
\begin{aligned}
\gamma_{1} \triangleq & \left(1-\delta_{1}\right) \exp \left(\int _ { ( n + l - 1 ) T } ^ { ( n + l ) T } \left(\frac{r\left(k_{0}-\overline{m_{1}}\right)}{k_{1}}-\left(b+a_{3}\right) M\right.\right. \\
& \left.\left.-\frac{a_{1}}{b_{1}}\left(v_{4}^{*}(t)+\eta_{1}\right)^{m}\right) \mathrm{~d} t\right)>1 .
\end{aligned}
$$

It is easy to prove that $x(t)<\overline{m_{1}}$ can not hold for all $t \geq 0$. Otherwise, we have

$$
\begin{cases}z^{\prime}(t)=z(t)\left(-m_{2}+\frac{e_{1} a_{1} x(t) z(t)^{m-1}}{b_{1}}+\frac{e_{2} a_{2} y(t) z(t)^{m-1}}{b_{2}}\right), & \\ \leq z(t)\left(-m_{2}+\frac{e_{1} a_{1} \overline{m_{1}} z(t)^{m-1}}{b_{1}}+\frac{e_{2} a_{2} M z(t)^{m-1}}{b_{2}}\right) \triangleq z(t) B, & t \neq n T, t \neq(n+l-1) T, \\ z\left(t^{+}\right)=\left(1-\delta_{3}\right) z(t), & t=(n+l-1) T, \\ z\left(t^{+}\right)=z(t)+p, & t=n T, \\ z\left(0^{+}\right)=z_{0} . & \end{cases}
$$

So we can obtain $v_{4}(t) \longrightarrow v_{4}^{*}(t)$ as $t \longrightarrow \infty\left(v_{4}(t)\right.$ is the solution of (49)) and $z(t) \leq v_{4}(t)$ :

$$
\begin{cases}v_{4}^{\prime}(t)=B v_{4}(t), & t \neq n T, t \neq(n+l-1) T \\ v_{4}\left(t^{+}\right)=\left(1-\delta_{3}\right) v_{4}(t), & t=(n+l-1) T \\ v_{4}\left(t^{+}\right)=v_{4}(t)+p, & t=n T \\ v_{4}\left(0^{+}\right)=z\left(0^{+}\right)>0, & \end{cases}
$$

and its periodic solution is as follows:

$$
v^{*}(t)= \begin{cases}\frac{p \exp (B(t-(n-1) T))}{1-\left(1-\delta_{3}\right) \exp (B T)}, & (n-1) T<t \leq(n+l-1) T, \\ \frac{p\left(1-\delta_{3}\right) \exp (B(t-(n-1) T))}{1-\left(1-\delta_{3}\right) \exp (B T)}, & (n+l-1) T<t \leq n T .\end{cases}
$$

We can easily get $z(t) \leq v_{4}(t)$ as $t \longrightarrow+\infty$ by using the comparison theorem. So there is a $T_{1}>0$, when $t>T_{1}$, $z(t) \leq v_{4}(t)<v_{4}^{*}(t)+\eta_{1}$. Therefore, we have

$$
\begin{cases}x^{\prime}(t) \geq x(t)\left(\frac{r\left(k_{0}-\overline{m_{1}}\right)}{k_{1}}-\left(b+a_{3}\right) M-\frac{a_{1}}{b_{1}}\left(v_{4}^{*}(t)+\eta_{1}\right)^{m}\right), & t \neq n T, t \neq(n+l-1) T, \\ x\left(t^{+}\right)=\left(1-\delta_{1}\right) x(t), & t=(n+l-1) T .\end{cases}
$$


$N_{1} \in N$ and $\left(N_{1}+l-1\right) T \geq T_{1}$. Integrating (51) on

$((n+l-1) T,(n+l) T], n \geq N_{1}$, we have

$$
\begin{aligned}
& x((n+l) T) \geq x\left((n+l-1) T^{+}\right) \exp \left(\int_{(n+l-1) T}^{(n+l) T}\left(\frac{r\left(k_{0}-\overline{m_{1}}\right)}{k_{1}}-\left(b+a_{3}\right) M-\frac{a_{1}}{b_{1}}\left(v_{4}^{*}(t)+\eta_{1}\right)^{m}\right) \mathrm{d} t\right) \\
& \quad=x((n+l-1) T)\left(1-\delta_{1}\right) \exp \left(\int_{(n+l-1) T}^{(n+l) T}\left(\frac{r\left(k_{0}-\overline{m_{1}}\right)}{k_{1}}-\left(b+a_{3}\right) M-\frac{a_{1}}{b_{1}}\left(v_{4}^{*}(t)+\eta_{1}\right)^{m}\right) \mathrm{d} t\right) \\
& \quad=x((n+l-1) T) \gamma_{1} .
\end{aligned}
$$

Some algebraic calculations of (52), which produces $x\left(\left(N_{1}+k\right) T\right) \geq x\left(\left(N_{1}\right) T\right) \gamma_{1}^{k} \longrightarrow \infty$ as $k \longrightarrow \infty$. Obviously, this is contradictory to the boundedness of $x(t)$. Therefore, there is a $t_{1}>0$ such that $x\left(t_{1}\right) \geq \overline{m_{1}}$.

Secondly, if $x(t), y(t), q(t) \geq \overline{m_{1}}$ for all $t \geq t_{1}$, then the proof of this theorem is completed. Otherwise, $x(t)>\overline{m_{1}}$ can not hold for all $t \geq t_{1}$. So we need to find out which solutions leave the set $\left\{X(t) \in R_{+}^{4}: x(t) \geq \overline{m_{1}}\right\}$, and let these solutions return to the set again. Let $t^{*}=\inf _{t \geq t_{1}}\left\{x(t)<\overline{m_{1}}\right\}$, which has the following cases:
Case 1: if $t^{*}=\left(n_{1}+l-1\right) T$, then $x(t) \geq \overline{m_{1}}$ and $y(t) \geq \overline{m_{1}}$ for $t \in\left[t_{1}, t^{*}\right)$ and $\left(1-\delta_{1}\right) \overline{m_{1}} \leq x\left(t^{*+}\right)$ $=\left(1-\delta_{1}\right) x\left(t^{*}\right)<\overline{m_{1}}$ and $\left(1-\delta_{2}\right) \overline{m_{1}} \leq y\left(t^{*+}\right)=$ $\left(1-\delta_{2}\right) y\left(t^{*}\right)<\overline{m_{1}}$. Choose $n_{2}, n_{3} \in N$ such that $\left(n_{2}-\right.$ 1) $T>\left(\ln \left(\eta_{1} / M+p\right) / B_{1}\right),\left(1-\delta_{1}\right)^{n_{2}} \gamma_{1}^{n_{3}} \quad \exp \left(\left(\gamma_{2} n_{2} T\right)\right.$ $\left.\gamma_{1}^{n_{3}}\right)>\left(1-\delta_{1}\right)^{n_{2}} \exp \left(\gamma_{2}\left(n_{2}+1\right) T\right) \gamma_{1}^{n_{3}}>1$, where $\gamma_{2}=$ $\left(r\left(k_{0}-\overline{m_{1}}\right) / k_{1}\right)-\left(b+a_{3}\right) M-\left(a_{1} / b_{1}\right) M^{m}<0$. Let $\bar{T}=$ $n_{2} T+n_{3} T$, and it is deduced that there must be a $t^{\prime} \in\left(t^{*}, t^{*}+\bar{T}\right)$ such that $x\left(t^{\prime}\right)>\overline{m_{1}}$; considering (49) with $v_{4}\left(n_{1} T^{+}\right)=x_{3}\left(n_{1} T^{+}\right)$, it is easy to get

$$
v_{4}(t)=\left\{\begin{array}{l}
\left(1-\delta_{3}\right)^{n-\left(n_{1}+l\right)}\left(z\left(n_{1} T^{+}\right)-\frac{p}{1-\left(1-\delta_{3}\right) \exp (B T)}\right) \exp \left(B\left(t-n_{1} T\right)\right)+v_{4}^{*}(t),(n-1) T<t \leq(n+l-1) T, \\
\left(1-\delta_{3}\right)^{n-\left(n_{1}+l-1\right)}\left(z\left(n_{1} T^{+}\right)-\frac{p}{1-\left(1-\delta_{3}\right) \exp \left(B_{1} T\right)}\right) \exp \left(B\left(t-n_{1} T\right)\right)+v_{4}^{*}(t),(n+l-1) T<t \leq n T,
\end{array}\right.
$$

and $n_{1}+1 \leq n \leq n_{1}+n_{2}+n_{3}+1$. So we can get $\mid v_{4}(t)-$ $v_{4}^{*}(t) \mid<(M+p) \exp \left(B\left(t-n_{1} T\right)\right)<\eta_{1}$ and $z(t) \leq v_{4}(t)$ $<v_{4}^{*}(t)+\eta_{1}$ for $t^{*}+n_{2} T \leq t \leq t^{*}+\bar{T}$, which indicate that (51) holds for $t^{*}+n_{2} T \leq t \leq t^{*}+\bar{T}$. We can obtain
$x\left(t^{*}+\bar{T}\right) \geq x\left(t^{*}+n_{2} T\right) \gamma_{1}^{n_{3}}$ through a discussion similar to the first step. From the system (8), we have

$$
\begin{cases}x^{\prime}(t) \geq x(t)\left(\frac{r\left(k_{0}-\overline{m_{1}}\right)}{k_{1}}-\left(b+a_{3}\right) M-\frac{a_{1}}{b_{1}} M^{m}\right)=\gamma_{2} x(t), t \neq n T, & t \neq(n+l-1) T, \\ x\left(t^{+}\right)=\left(1-\delta_{1}\right) x(t), & t=(n+l-1) T .\end{cases}
$$

Integrating (54) on $t \in\left[t^{*}, t^{*}+n_{2} T\right]$, then we get $x\left(t^{*}+n_{2} T\right) \geq \overline{m_{1}}\left(1-\delta_{1}\right)^{n_{2}} \exp \left(\gamma_{2} n_{2} T\right)$ and $x\left(t^{*}+\bar{T}\right)$ $\geq \overline{m_{1}}\left(1-\delta_{1}\right)^{n_{2}} \gamma_{1}^{n_{3}} \exp \left(\gamma_{2} n_{2} T\right)>\overline{m_{1}}$; it is a contradiction with $x(t)<\overline{m_{1}}$. So $x(t)>\overline{m_{1}}$ holds for all $t \in\left(t_{1}, t^{*}\right)$. Let $\bar{t}=\inf _{t \geq t^{*}}\left\{x(t) \geq \overline{m_{1}}\right\}$, then we can obtain $x(t) \leq \overline{m_{1}}, x(\bar{t})=\overline{m_{1}}$ and $x(t) \geq \overline{m_{1}}\left(1-\delta_{1}\right)^{n_{2}+n_{3}}$ $\exp \left(\left(n_{2}+n_{3}\right) \gamma_{2} T\right) \triangleq m_{1}$ for $t^{*}<t<\bar{t}$. Then, we derive that $x(t) \geq m_{1}$ holds for $t^{*}<t<\bar{t}$. In addition, for $t>\bar{t}$, since $x(\bar{t}) \geq \overline{m_{1}}$, the same discussion can be continued.

Case 2: the case of $t^{*}=\left(n_{1}+l-1\right) T$ is discussed in Case 1. Now, assume that $t^{*} \neq\left(n_{1}+l-1\right) T$, which produces $x(t) \geq \overline{m_{1}}$ for $t_{1} \leq t<t^{*}$ and $x_{1}\left(t^{*}\right)=\overline{m_{1}}$. If $t^{*} \in\left(\left(n_{1}^{\prime}+l-1\right) T,\left(n_{1}^{\prime}+l\right) T\right), n_{1}^{\prime} \in N$, which have two possible cases for $t \in\left(t^{*},\left(n_{1}^{\prime}+l\right) T\right)$. 
Case 2(a): for all $t \in\left(t^{*},\left(n_{1}^{\prime}+l\right) T\right), x(t) \leq \overline{m_{1}}$ is valid. By the same discussion with case 1 , which exists $t_{1}^{\prime} \in\left[\left(n_{1}^{\prime}+\right.\right.$ $\left.l) T,\left(n_{1}^{\prime}+l\right) T+\bar{T}\right]$ such that $x\left(t_{1}^{\prime}\right)>\overline{m_{1}}$; in order to simplify the proof, we will not elaborate on it. Let $\widetilde{t}=\inf _{t \geq t^{*}}\left\{x(t)>\overline{m_{1}}\right\}$, then we have $x(\widetilde{t}) \leq \overline{m_{1}}$ for $t^{*} \leq t<\tilde{t}$. We get $\quad x(t) \geq \overline{m_{1}}\left(1-\delta_{1}\right)^{n_{2}+n_{3}+1} \exp$ $\left(\left(n_{2}+n_{3}\right) \gamma_{2} T\right) \triangleq m_{1}$ and $x(t) \geq m_{1}$ for $t \in\left(t^{*}, \tilde{t}\right)$. For $t>\widetilde{t}$, the same arguments can be followed since $x_{1}(\widetilde{t}) \geq \overline{m_{1}}$.

Case 2(b): there exists $t^{*}<t<\left(n_{1}^{\prime}+l\right) T$ such that $x(t)>\overline{m_{1}}$. Let $\widetilde{t}=\inf _{t>t^{*}}\left\{x(t)>\overline{m_{1}}\right\}$, then $x(\widetilde{t}) \leq \overline{m_{1}}$ for $t \in\left(t^{*}, \widetilde{t}\right)$ and $x_{1}(\widetilde{t})=\overline{m_{1}}$. When $t \in\left(t^{*}, \widetilde{t}\right)$, (54) holds. Integrating (54) on $\left(t^{*}, \widetilde{t}\right)$, we obtain $x(t) \geq x\left(t^{*}\right) \exp \left(\gamma_{2}\left(t-t^{*}\right)\right) \geq \overline{m_{1}} \exp \left(\gamma_{2} T\right)>m_{1}$. Since $x(t) \geq \overline{m_{1}}$ for $t>t^{*}$, the same arguments can be continued. Hence, $x(t) \geq m_{1}$ for all $t \geq \tilde{t}$.

To sum up all the above discussions, we derive that $x(t) \geq m_{1}$ holds for all $t \geq \tilde{t}$. Using similar methods for $y(t)$, we can get $y(t) \geq m_{2}$ for all $t \geq t_{2}$. Obviously, $q(t) \geq m_{1}$ for all $t>\widetilde{t}_{1}$. Let $m=\max \left(m_{1}, m_{2}, m_{3}\right)$; by Theorem 1 and Definition 2, we obtain that $m<x(t), y(t), z(t), q(t)<M$. So, the system (8) proved to be permanent.

\section{Extension of Theoretical Results}

In this section, we can see that Section 3 of this paper can be extended to the results of the literature [26].

From Theorem 2, we know if

$$
\begin{aligned}
& \frac{r k_{0} T}{k_{1}}+\frac{a_{1}}{c_{1} m m_{2}}\left(A_{1}+A_{2}\right)<\ln \frac{1}{1-\delta_{1}}, \\
& -m_{1} T+\frac{a_{2}}{c_{2} m m_{2}}\left(B_{1}+B_{2}\right)<\ln \frac{1}{1-\delta_{2}},
\end{aligned}
$$

hold, then the eradication periodic solution $\left(0,0, z^{*}(t), 0\right)$ of species of $x(t)$ and $y(t)$ is globally asymptotically stable, where $A, A_{1}, A_{2}, B_{1}$, and $B_{2}$ are given in Theorem 2 . If $m=1, \delta_{i}(i=1,2,3)=0, l=1$, inequality (55) is equivalent to

$$
\frac{r k_{0} T}{k_{1}}+\frac{a_{1}}{m_{2} c_{1}} \ln \left(1-\frac{c_{1} p\left[1-\exp \left(-m_{2} T\right)\right]}{b_{1}\left[1-\exp \left(-m_{2} T\right)\right]+c_{1} p}\right)<0 .
$$

In addition, inequality (56) is equivalent to

$$
-m_{1} T+\frac{a_{2}}{m_{2} c_{2}} \ln \left(1-\frac{c_{2} p\left[1-\exp \left(-m_{2} T\right)\right]}{b_{2}\left[1-\exp \left(-m_{2} T\right)\right]+c_{2} p}\right)<0 .
$$

Remark 2. Theorem 2.1 of the literature [26] can be obtained by combining inequalities (57) and inequalities (58).

From the above discussion, the following corollary can be obtained.

Corollary 1. If $m=1, \delta_{i}(i=1,2,3)=0, l=1$, then the eradication periodic solution $\left(0,0, z^{*}(t), 0\right)$ of species of $x(t)$ and $y(t)$ of system (8) is locally asymptotically stable if

$$
\left\{\begin{array}{l}
\frac{r k_{0} T}{k_{1}}+\frac{a_{1}}{m_{2} c_{1}} \ln \left(1-\frac{c_{1} p\left[1-\exp \left(-m_{2} T\right)\right]}{b_{1}\left[1-\exp \left(-m_{2} T\right)\right]+c_{1} p}\right)<0, \\
-m_{1} T+\frac{a_{2}}{m_{2} c_{2}} \ln \left(1-\frac{c_{2} p\left[1-\exp \left(-m_{2} T\right)\right]}{b_{2}\left[1-\exp \left(-m_{2} T\right)\right]+c_{2} p}\right)<0 .
\end{array}\right.
$$

From Theorem 3, we know that if

$$
\left\{\begin{array}{l}
\frac{r k_{0} T}{k_{1}}+\frac{a_{1}}{c_{1} m m_{2}}\left(A_{1}+A_{2}\right)>\ln \frac{1}{1-\delta_{1}}, \\
-m_{1} T+\frac{a_{2}}{c_{2} m m_{2}}\left(B_{1}+B_{2}\right)>\ln \frac{1}{1-\delta_{2}},
\end{array}\right.
$$

hold, then system (8) is permanent. Similar to the discussion of inequality (55)-(58), we can obtain the following corollary.

Corollary 2. If $m=1, \delta_{i}(i=1,2,3)=0$, and $l=1$, the system (8) is permanent provided that

$$
\left\{\begin{array}{l}
\frac{r k_{0} T}{k_{1}}+\frac{a_{1}}{m_{2} c_{1}} \ln \left(1-\frac{c_{1} p\left[1-\exp \left(-m_{2} T\right)\right]}{b_{1}\left[1-\exp \left(-m_{2} T\right)\right]+c_{1} p}\right)>0, \\
-m_{1} T+\frac{a_{2}}{m_{2} c_{2}} \ln \left(1-\frac{c_{2} p\left[1-\exp \left(-m_{2} T\right)\right]}{b_{2}\left[1-\exp \left(-m_{2} T\right)\right]+c_{2} p}\right)>0 .
\end{array}\right.
$$

Remark 3. Corollary 1 and Corollary 2 are the same as Theorem 2.1 and Theorem 2.4 of the literature [26], respectively. Our theoretical results generalize the results of the literature [26].

Remark 4. After calculation and comparison, we can know that there are some mistakes in the form of Theorem 2.1, Theorem 2.3, and Theorem 2.4 of the literature [26].

\section{Examples and Simulations}

The effect of numerical experiments on this system is to help us visually observe the dynamic behavior of species changing with parameters, so some dynamical properties of the system (8) are showed by numerical simulation in this section, which is based on the parameters of the system in accordance with actual biological significance.

Firstly, let $r=0.6, k_{1}=20, k_{0}=15, a_{1}=0.5, a_{2}=0.5$, $a_{3}=0.5, e_{1}=0.8, e_{2}=0.85, c_{1}=0.6, \quad c_{2}=0.5, b_{1}=1.5, b_{2}=$ $0.75, m_{1}=0.1, m_{2}=0.15, \quad d=0.9, b=0.15, a=1.8, l=0.7$, $\delta_{1}=0.65, \delta_{2}=0.45, \delta_{3}=0.2, m=0.92$, and $p=10$. By verification, these parameters satisfy biological significance and the conditions of Theorem 2, so the eradication periodic solution $\left(0,0, z^{*}(t), 0\right)$ of species $x(t)$ and $y(t)$ is globally asymptotically stable under this numerical scenario. By the numerical experiment, Figures 1(a)-1(e) and Figure 2 show that the system is in asymptotic stability. Figures $1(\mathrm{a})-1(\mathrm{e})$ illustrate the existence of the solution $\left(0,0, z^{*}(t), 0\right)$, and the dynamic behavior of species $x(t)$ and $y(t)$ with different initial values is described in Figure 2. In addition, let $m=0.61$ while keeping 


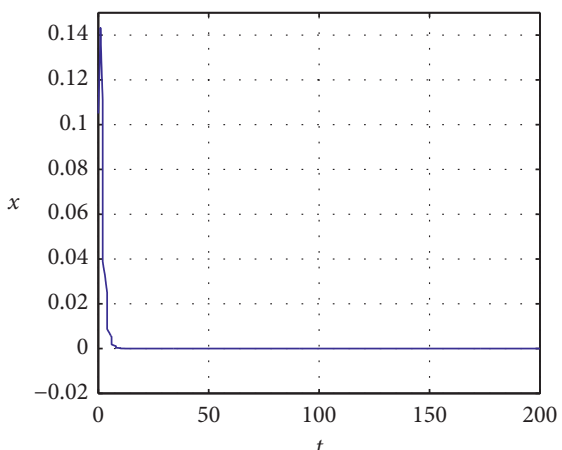

(a)
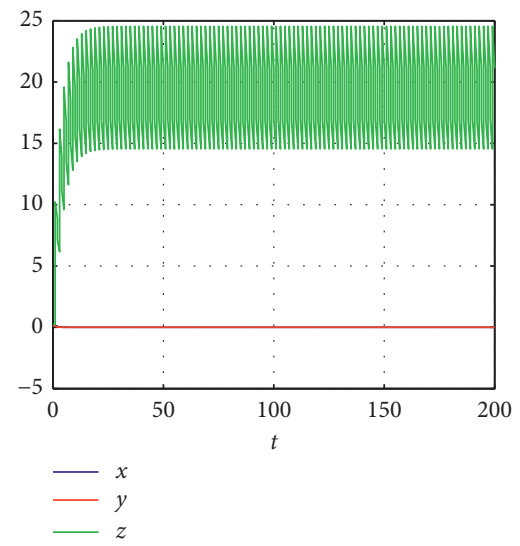

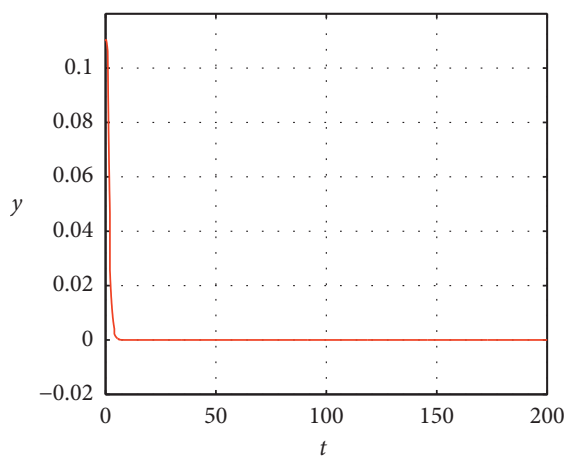

(b)

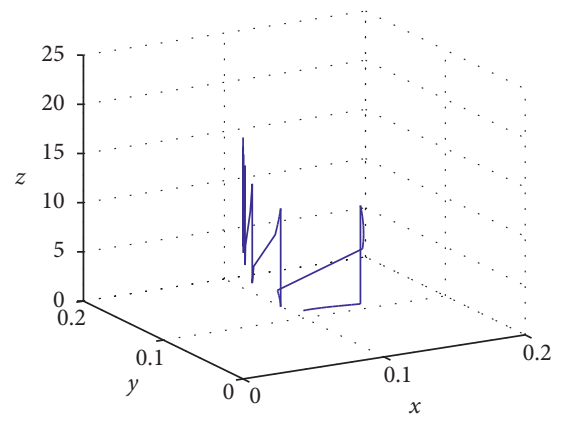

(e)

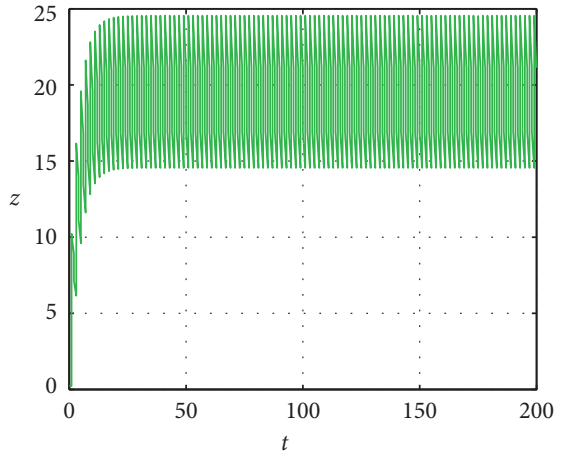

(c)

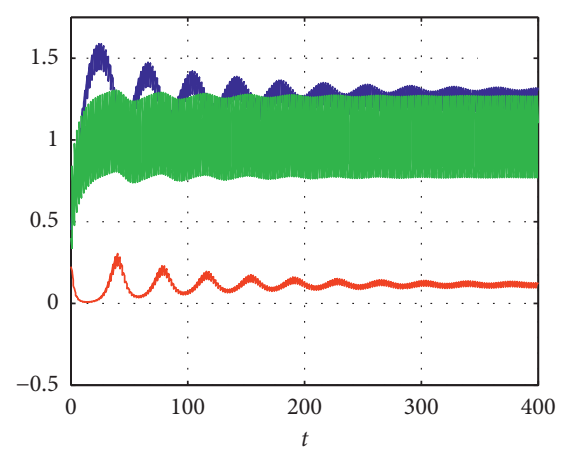

$x$
$-y$
$-z$

(d)

(f)

Figure 1: The eradication periodic solution $\left(0,0, z^{*}(t), 0\right)$ of species $x(t)$ and $y(t)$ with $x(0)=0.3, y(0)=0.2, z(0)=0.3$, and $q(0)=0.3$. (a)-(c) Time series of species $x(t), y(t)$, and $z(t)$, respectively; (d) time series of three species $x(t), y(t)$, and $z(t)$, which are in the same quadrant; (e) phase portrait of $x(t), y(t)$, and $z(t)$; (f) a special case of dynamic behaviors as $m=0.61$.

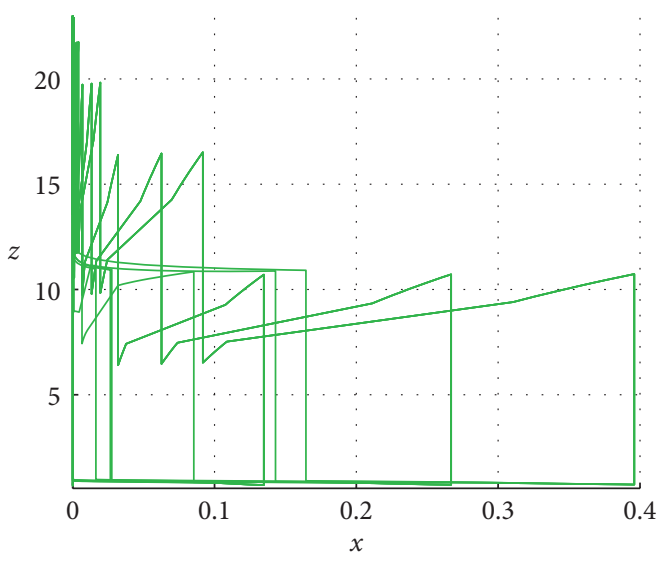

(a)

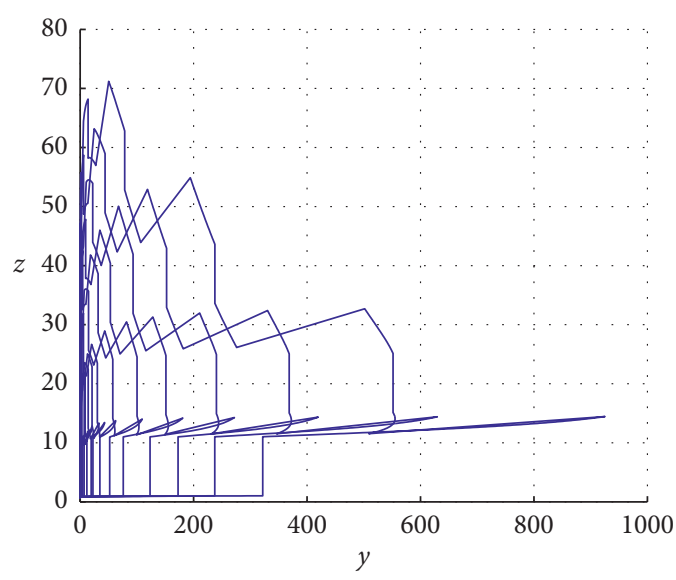

(b)

FIgURE 2: Dynamic behavior of $\left(0,0, z^{*}(t), 0\right)$ with different initial values, where the values of parameters are consistent with the settings in Figures 1 (a) and 1(b) phase portrait of species $x(t)$ and $z(t)$ and $y(t)$ and $z(t)$ for different initial values, respectively.

other parameters unchanged, we will be surprised to find that the system becomes persistent (see Figure 1(f)) through numerical experiments, so Figure 1(f) shows that the parameter $m$ has a certain influence on the dynamic properties of the system (8).

Secondly, let $r=0.6, k_{1}=19, k_{0}=15, a_{1}=0.8, a_{2}=0.9$, $a_{3}=0.2, e_{1}=0.33, e_{2}=0.1, c_{1}=0.6, \quad c_{2}=0.33, b_{1}=1.5$, $b_{2}=0.75, m_{1}=0.25, m_{2}=0.25, d=0.9, b=0.119, a=1.8$, $l=0.6, \delta_{1}=0.1, \delta_{2}=0.35, \delta_{3}=0.25, m=0.9$, and $p=0.5$.

By verification, these parameters satisfy biological significance and the conditions of Theorem 3, so the system (8) is permanent under this numerical scenario. By numerical simulation, the same conclusion is verified (see Figures 3(a)-3(d)). Similarly, let $m=0.3$ while keeping other 


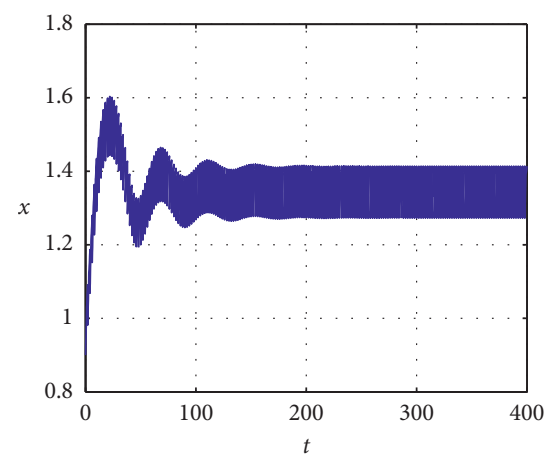

(a)

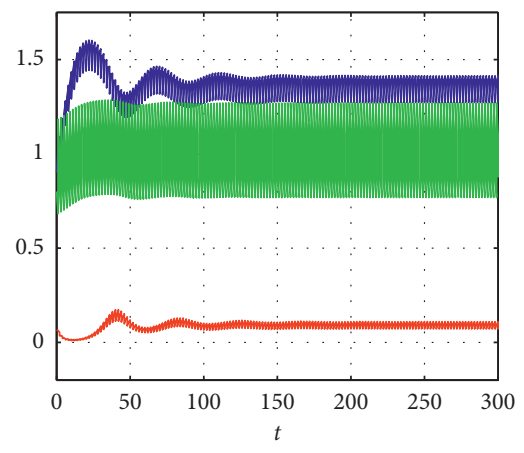

$x$
$-y$
$-z$

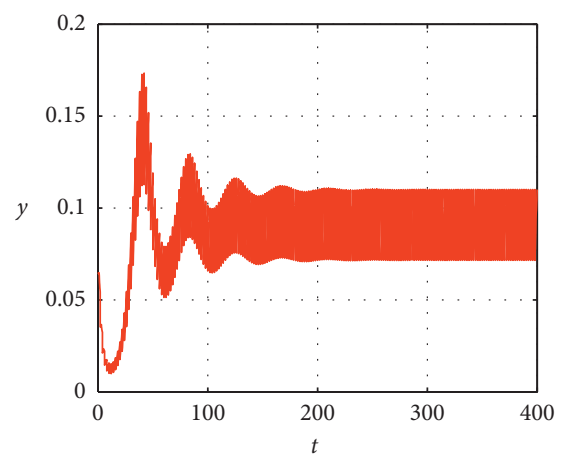

(b)

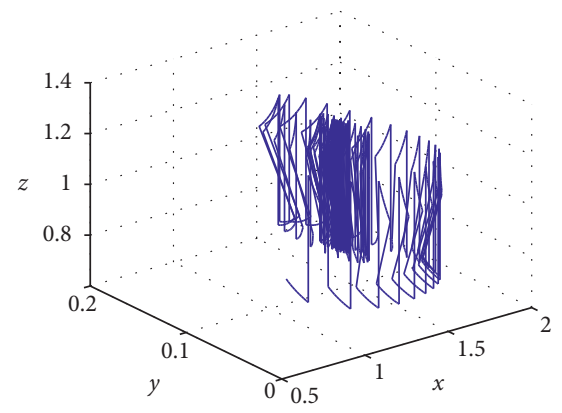

(e)

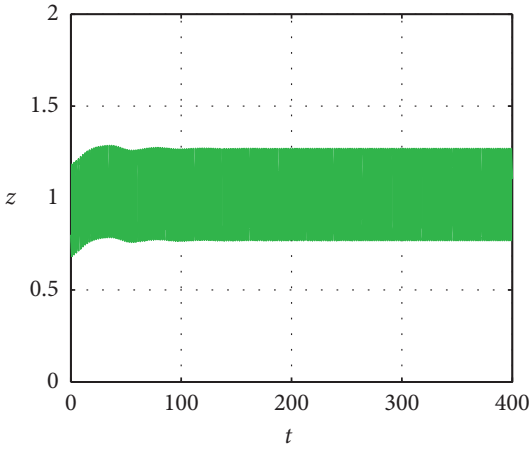

(c)
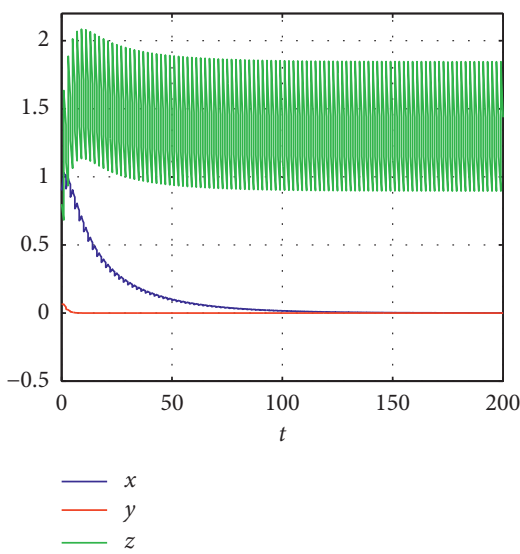

(d)

Figure 3: (a-c) Time series of persistence of $x(t), y(t)$, and $z(t)$, respectively, which initial values of $x(0)=1, y(0)=0.1, z(0)=1$, and $q(0)=1 ;(\mathrm{d})$ time series of three species $x(t), y(t)$, and $z(t)$ in the same quadrant; (e) phase portrait of $x(t), y(t)$, and $z(t)$; (f) special case of dynamic behaviors as $m=0.34$.

parameters unchanged, we will be surprised to find that species $x(t)$ and $y(t)$ tend to extinct (see Figure 3(f)); i.e., the system has changed from a persistent situation to an extinct one, so we can see that the influence of parameter $m$ on the system is effective.

Thirdly, by numerical analysis of bifurcation diagrams of impulsive period $T$, the dynamic properties of the system (8) can be showed. Let $x(0)=2, y(0)=2, z(0)=3$, and $q(0)=1 . \quad r=0.6, k_{1}=20, k_{0}=15, a_{1}=0.8, a_{2}=0.9, a_{3}=$ $0.2, e_{1}=0.8, e_{2}=0.1, c_{1}=0.6, c_{2}=0.5, b_{1}=0.25, b_{2}=0.75$, $m_{1}=0.1, m_{2}=0.15, d=0.25, b=0.15, a=1.8, l=0.7, \delta_{1}=$ $0.65, \delta_{2}=0.45, \delta_{3}=0.25, m=0.9$, and $p=0.5$. The bifurcation diagrams of impulsive period $T$ is presented in Figure 4, whose range of values is [17, 23]. From Figures 4(a)-4(c), we can observe that impulsive period $T$ has a great influence on the system. Since their bifurcation diagrams show many of the same phenomena, we will only explain the bifurcation diagram of $x(t)$ in detail here. Figures 5 and 6 are detailed demonstrations of Figure 4(a). When $T$ is small, the bifurcation diagram exhibits periodic oscillations of the system; for example, it has a periodic oscillation as $T=16.5$ (see Figure 6(a)). When $T=17.85,19.68,20.46$, and 20.62 , bifurcation occurs, respectively. More concretely, if $T>17.85$, we can see from Figure 5(a) that the phenomenon of bifurcation diagram changes from period-doubling bifurcations' cascade to cascade; if $T>19.68,4 T$ period solution occurs (see Figure 5(a)), and a example is showed in Figure 6(b) $(T=19.7)$; if $T>20.46,8 T$ period solution appears (see Figure 5(a)), and a example is showed in Figure 6(c) $(T=20.51)$; if $T>20.62,16 T$ period solution occurs(see Figure 5(a)). As $T$ increased by more than 20.66, the system was in "crisis." Period bifurcation reappears when $T>21.22$ (see Figure 5(b)). In the neighborhood of $T=22.1$, symmetry-breaking bifurcations occur after period bifurcation (see Figure 5(c)), which is a special simple bifurcation that produces multiple steady states. When $T=22.61$, a typical chaotic oscillation appears (see Figure 5(c)); after this situation, chaos appears such as Figure $6(\mathrm{~d})(T=22.8)$. That is to say, when $T$ is less than 17.85 , the harvesting and release of the system is frequent; when $17.85<T<20.66$, period bifurcation appears; if $20.66<T<23$, the dynamical properties of the system (8) become very complex; that is, the development of the system is unpredictable. All these results indicate that impulsive period $T$ has a serious effect on the dynamic behavior of the system.

Fourthly, we explore the bifurcation diagram of impulsive release $p$. Let $T=0.5$, while other parameters are consistent with Figure 4, whose range of values is $[0,2.5]$ (see Figure 7). As we can see from Figure 5, if the amount of release $p$ is small, the system (8) has periodic oscillations such as $p=0.23$ (see Figure 8(a)). If the releasing amount $p$ 


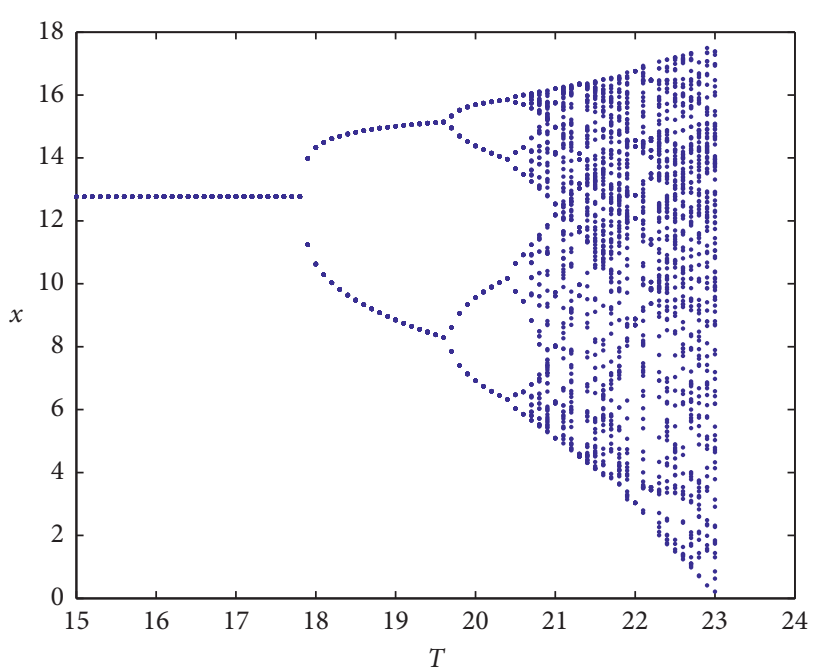

(a)

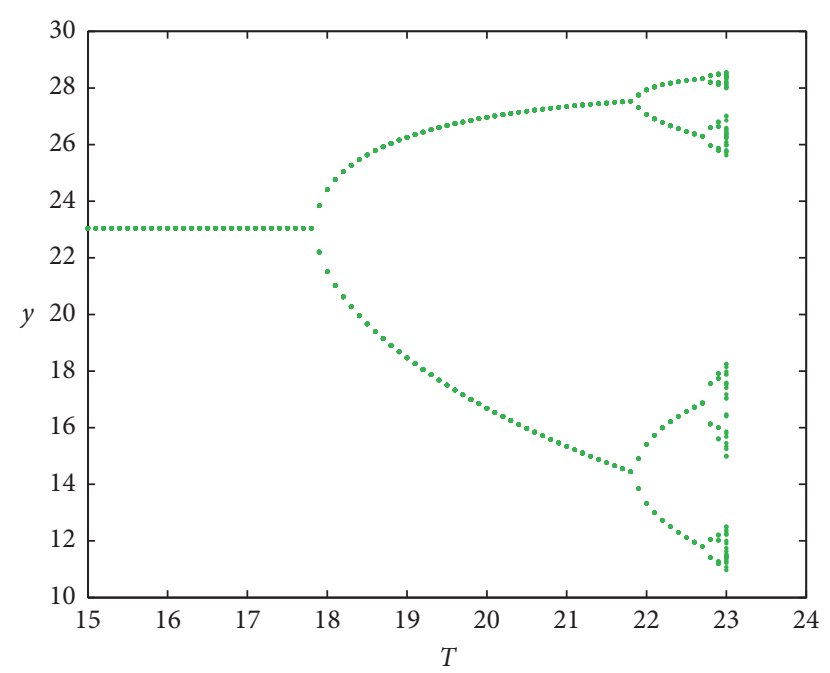

(b)

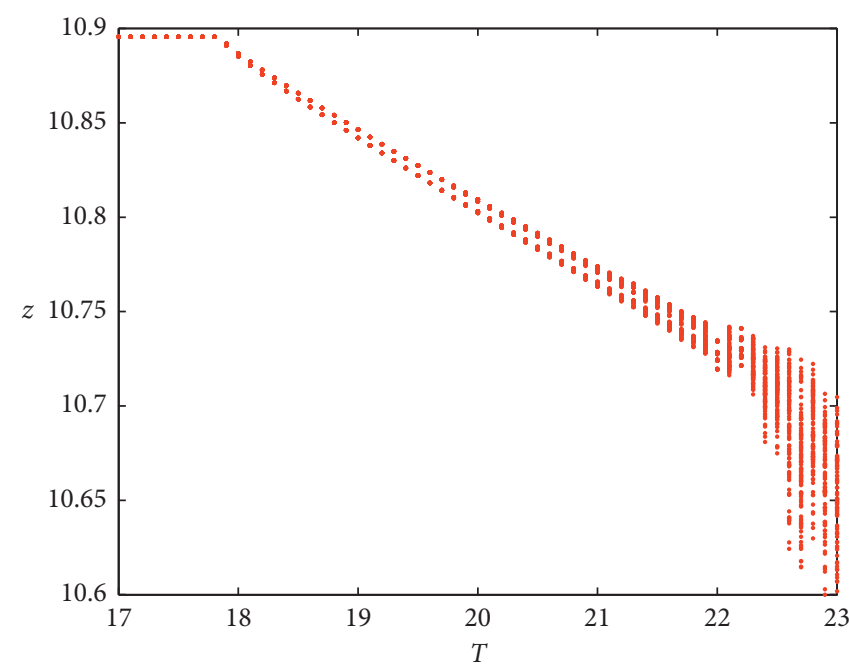

(c)

Figure 4: Bifurcation diagrams of parameter $(T)$. (a-c) Bifurcation diagram of $x(t), y(t)$, and $z(t)$, respectively.

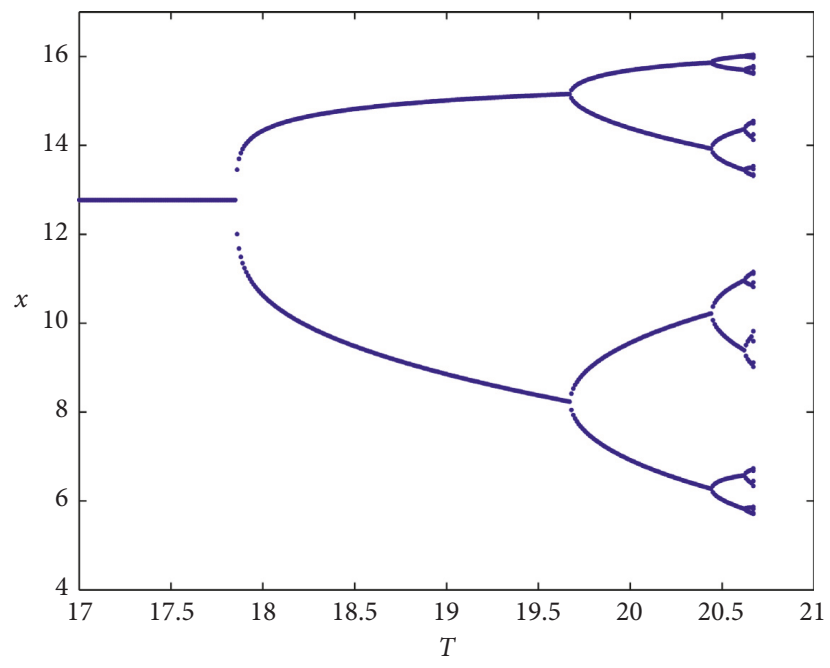

(a)

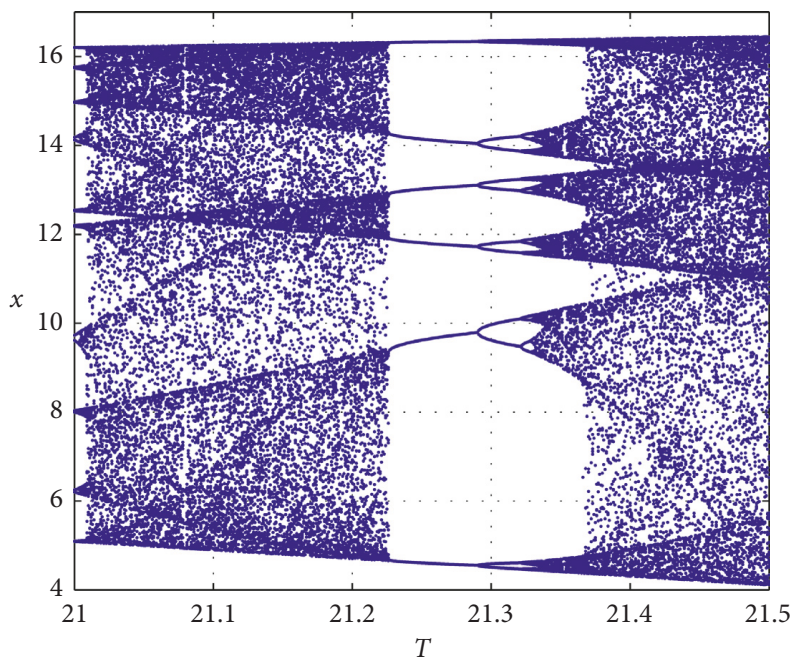

(b)

Figure 5: Continued. 


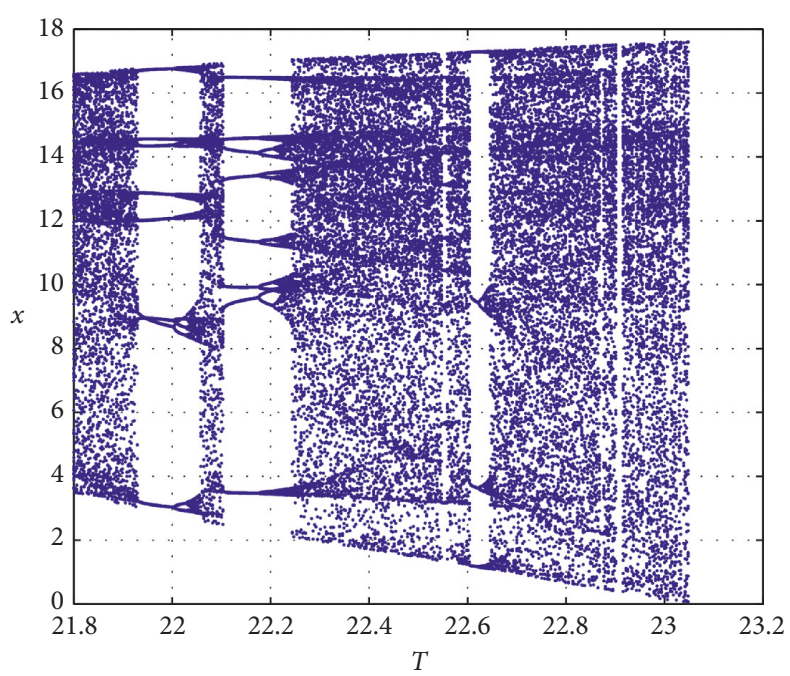

(c)

Figure 5: The detailed demonstrations of Figure 4(a). (a, b) The phenomenon of period bifurcation. (c) The phenomenon of crisis, symmetry-breaking bifurcations, and chaotic oscillation.

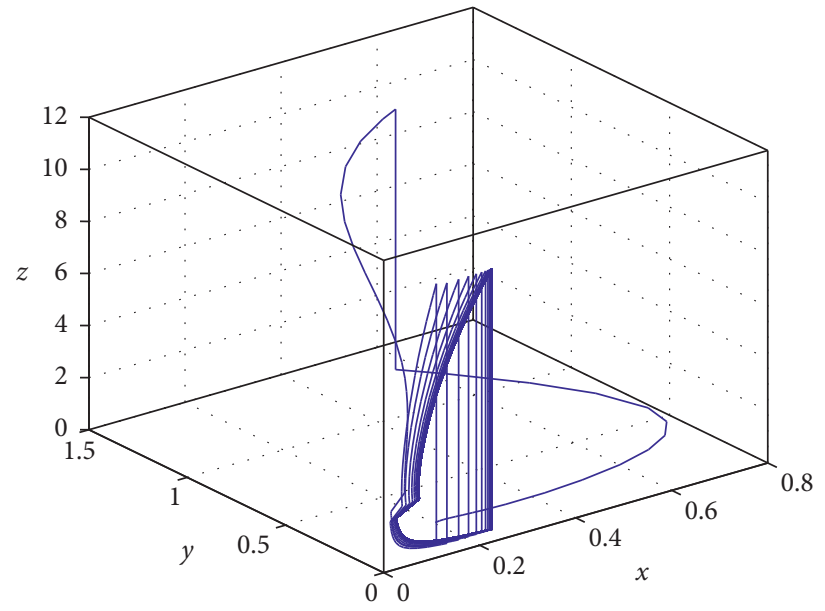

(a)

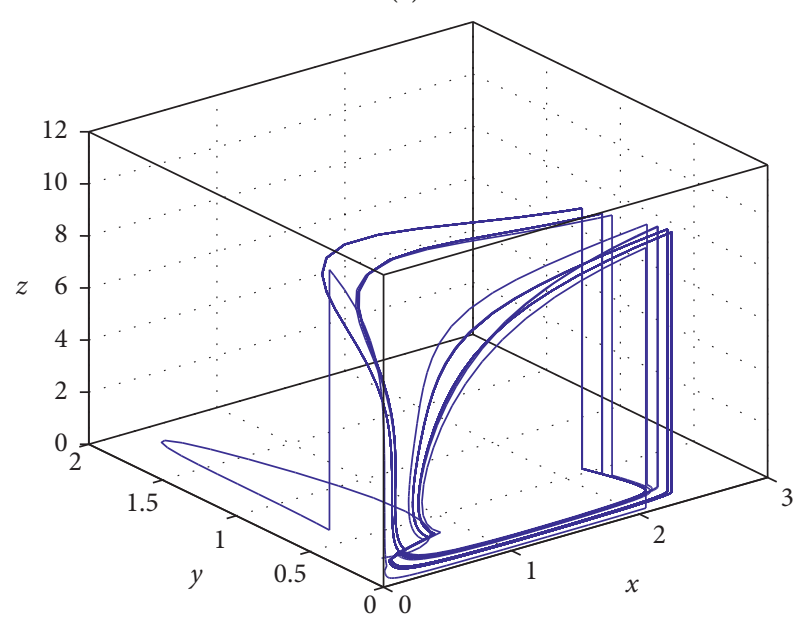

(c)

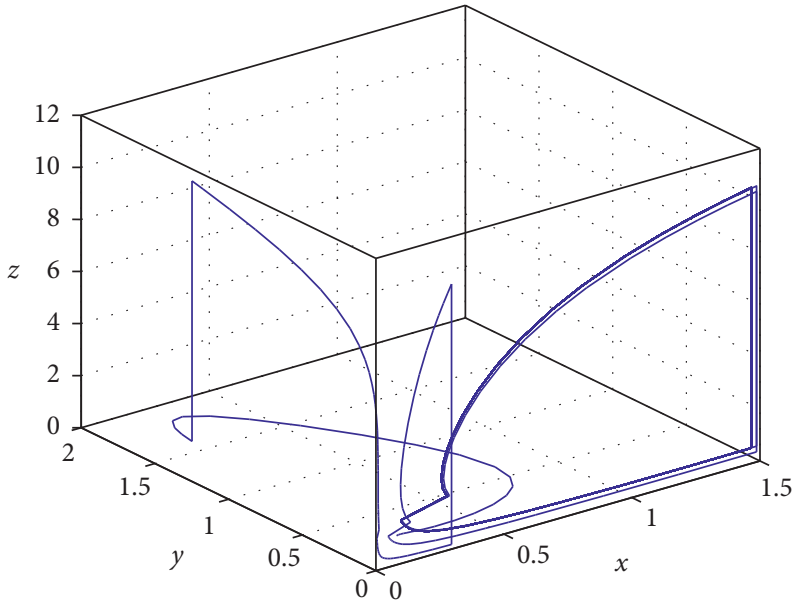

(b)

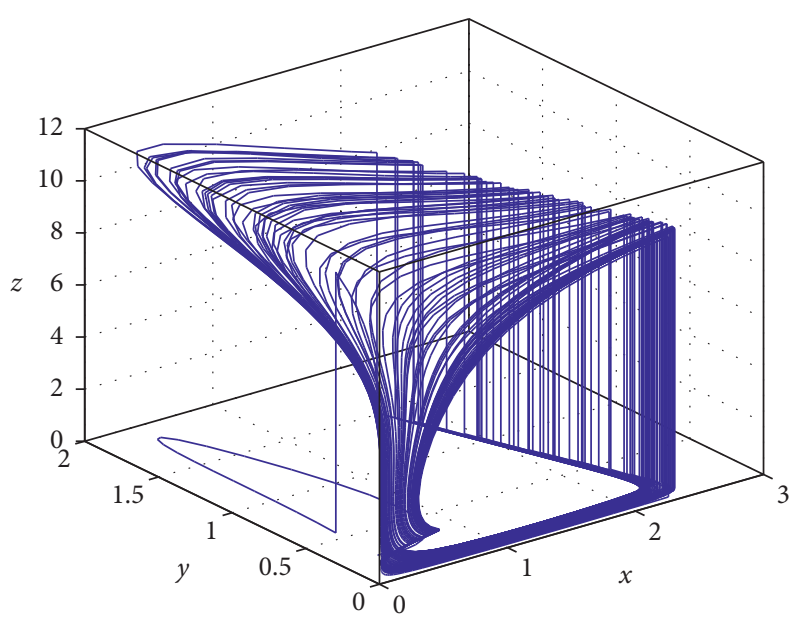

(d)

Figure 6: (a) Periodic oscillation when $T=16.5$; (b) $4 T$ period solution when $p=19.7$; (c) $8 T$ period solution when $p=20.51$; (d) chaos when $T=22.8$. 


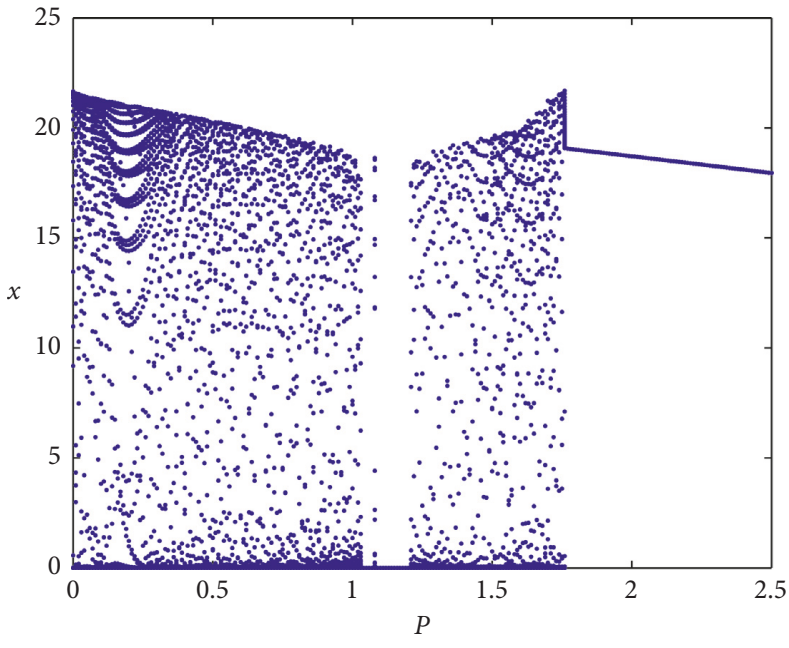

(a)

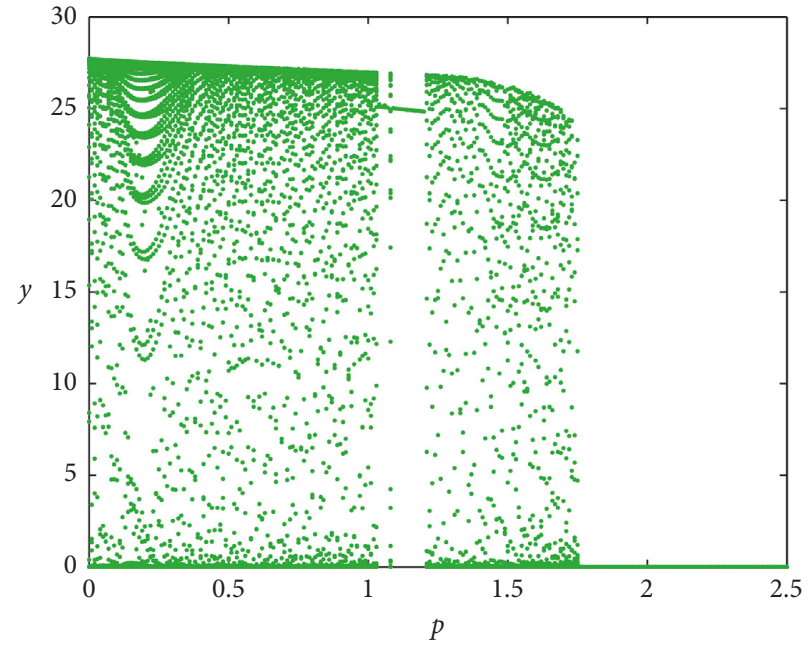

(b)

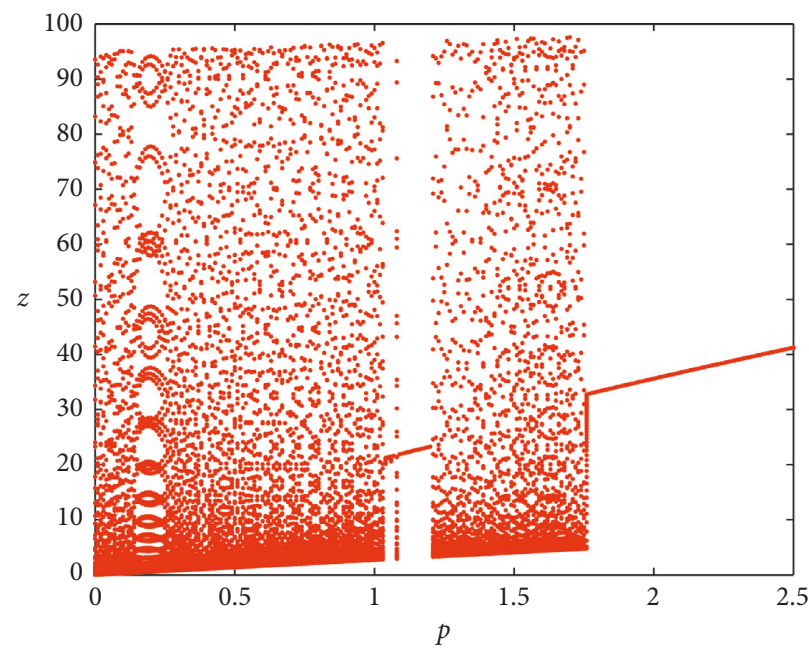

(c)

Figure 7: $(\mathrm{a}-\mathrm{c})$ The bifurcation diagrams of impulsive release $p$ of $x(t), y(t)$, and $z(t)$, respectively, with initial values $(1,1,1,1)$ and on $[0$, 2.5].

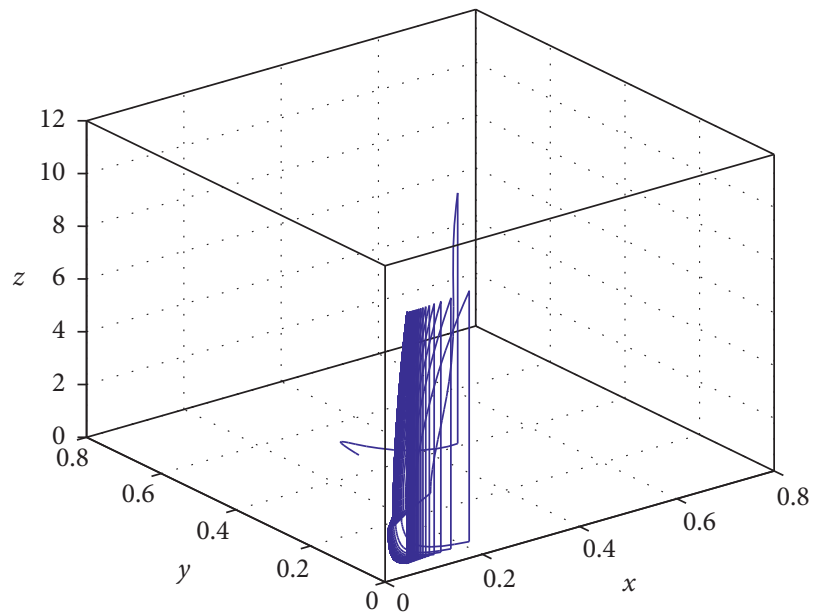

(a)

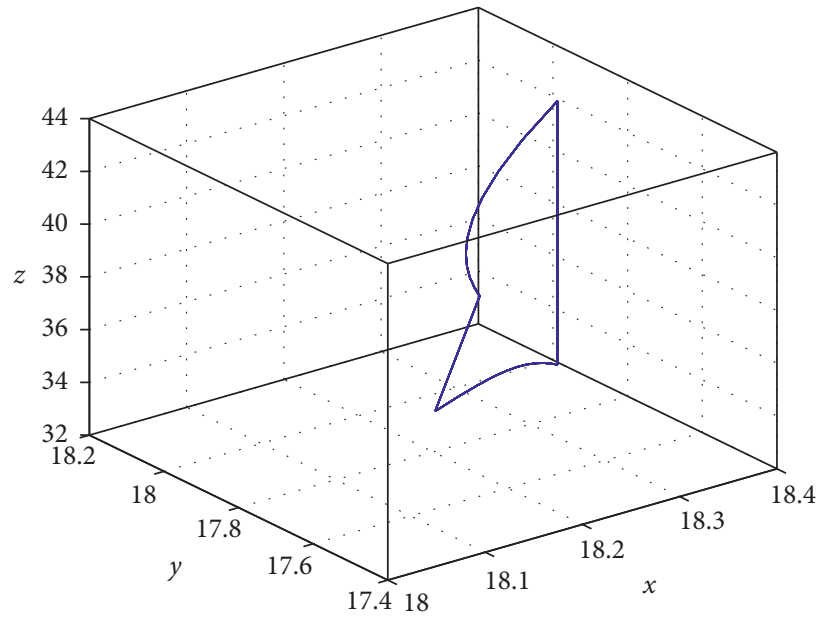

(b)

Figure 8: Continued. 


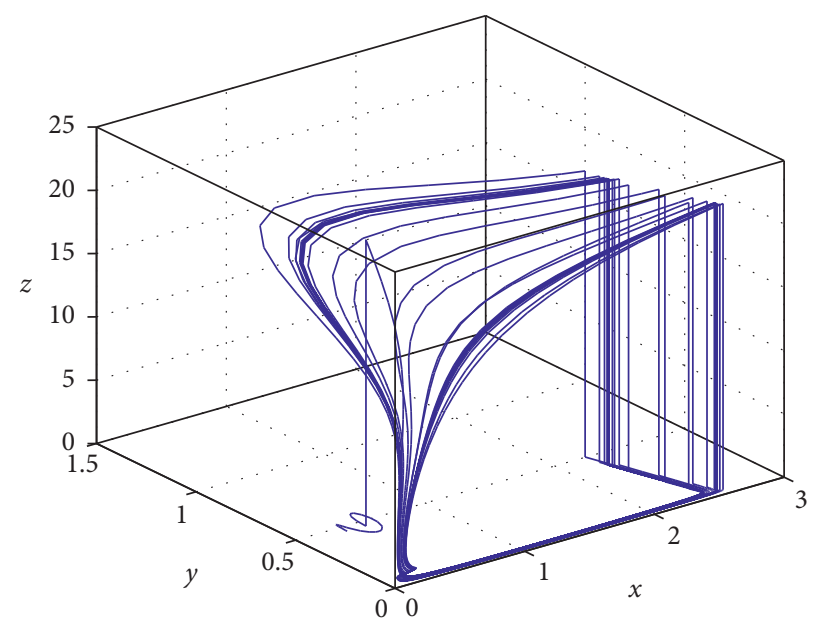

(c)

FIgURe 8: (a) Periodic oscillations when $p=0.23$; (b) $1 T$-period solution with $p=1.12$; (c) chaos with $p=0.65$.

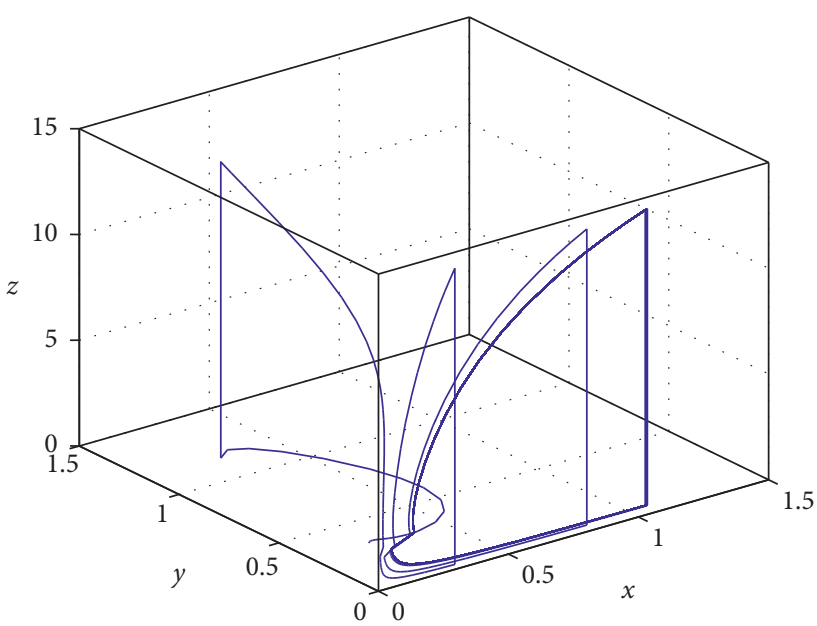

(a)

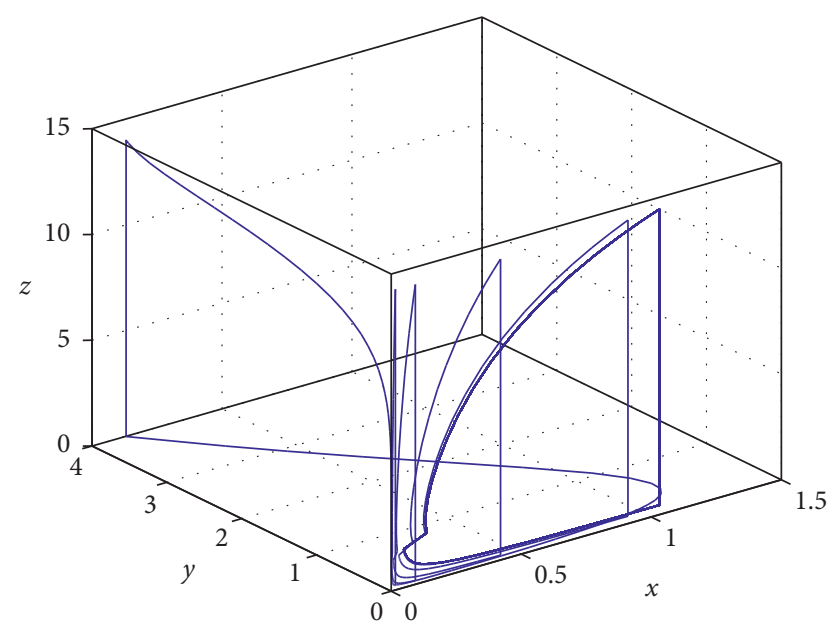

(b)

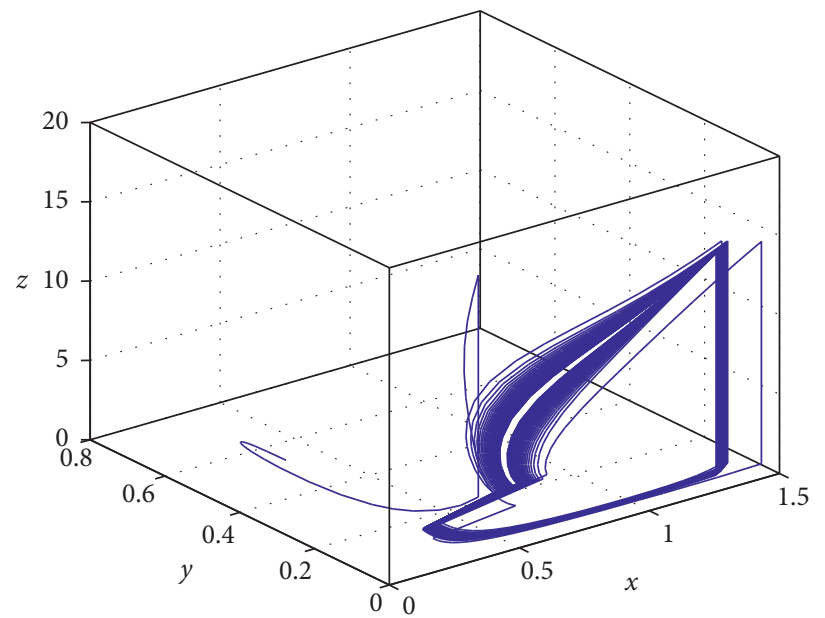

(c)

FiguRE 9: The value of parameter $m$ in Figure 8(b) is changed: (a) $4 T$-period solution when $m=0.35$; (b) $6 T$-period solution when $m=0.31$; (c) periodic oscillation when $m=0.95$. 


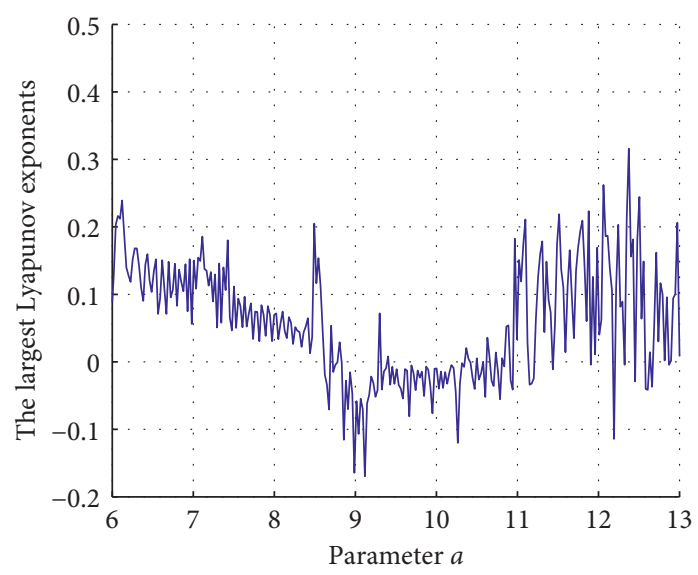

(a)

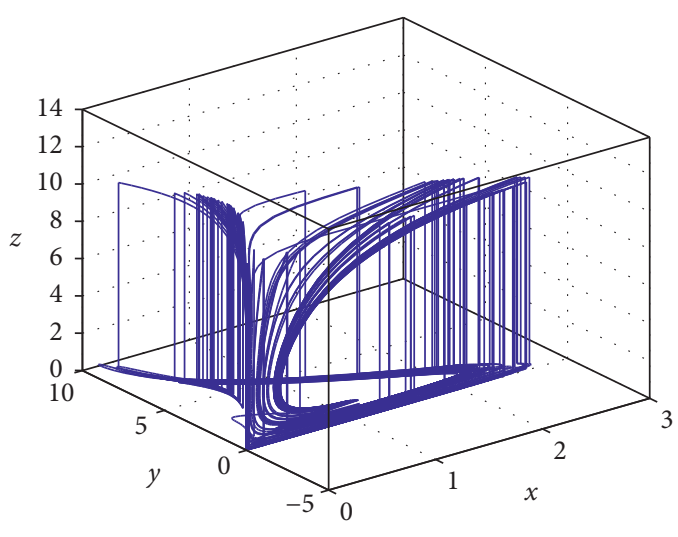

(b)

Figure 10: (a) The largest Lyapunov exponents of the system (8) with varying $a$ in $[6,13]$; (b) a strange attractor when $a=1.72$.

of predator $z(t)$ is comparatively large, then species $x(t)$ and $y(t)$ will die out. From Figure 7(b), we can see that predator $y(t)$ will die out when $T=1.77$, the number of $x(t)$ drops rapidly to zero when $p>27.25$, and this phenomenon shows the role of $z(t)$ as an advanced predator, predator $z(t)$, control predator $y(t)$, and prey $x(t)$. If the release amount $p$ is appropriate, there will be abundant phenomena in the system (8); for example, it produces chaos (see Figure 8(c)) when $p=0.65$, and it has $1 T$-period solution (see Figure 8(b)) when $p=1.12$. Furthermore, if the value of parameter $m$ in Figure 8(b) is changed, different dynamic properties can be obtained such as a $4 T$-period solution when $m=0.35$ (Figure 9(a)), a $6 T$-period solution when $m=0.31$ (see Figure 9(b)), and a phenomenon of periodic oscillation when $m=0.95$ (see Figure 9(c)). All these results show that the amount of impulsive release $p$ and mutual interference of predator $m$ produces complex dynamic behavior to the system (8).

Finally, the effect of distributed time delay $a$ on the system (8) is explored. Several recent experiments have become deterministic evidence for chaos, and the results of these literatures show that the study of chaos is very meaningful [37-39]. Among them, the largest Lyapunov exponents provide a very effective diagnosis for the chaotic system, and the largest Lyapunov exponent is the average change rate of the two trajectories which are close in phase space as time goes on by exponential separation or polymerization. In any system, as long as there is a largest Lyapunov exponent greater than zero, it considers the system to be a chaotic system [38]. Rosenstein et al. [39] introduces the general method and process of calculating largest Lyapunov exponents. The corresponding largest Lyapunov exponents of $a$ of system (8) is calculated and output in Figure 10(a), and an example of a strange attractor is given when $a=1.72$ (Figure 10(b)). All these results show that distributed time delay $a$ has an important effect on the system.

\section{Conclusion}

The dynamical analysis of a complex predator-prey system with mutual interference and the effect of time delay is presented in this paper, and numerical experiments of corresponding theoretical analysis are also presented. The periodic solution $\left(0,0, z^{*}(t), 0\right)$ is global asymptotically stable, which has been proved in Theorem 2 . The persistence of system (8) was proved in Theorem 3. As special cases, Corollary 1 and Corollary 2 generalize the theorems in the literature [26], and we have obtained more abundant theoretical results and numerical experiments.

After theoretical analysis and numerical simulation, we can know that impulsive period $T$, release amount $p$, mutual interference parameters $m$, and distributed time delay $a$ can produce different dynamical properties for system (8). For example, the solution $\left(0,0, z^{*}(t), 0\right)$ and the persistence of system (8) are given in Figures 1 and 3, respectively. Specially, the dynamical effect of mutual interference parameters $m$ is investigated. From Figures 1(d), 1(f), 3(d), 3(f), 8(b), and 9, we can know that different values of $m$ can produce different dynamical behaviors with the system (8), so it is necessary for us to study the influence of mutual interference in such a system.

In addition, the figure of largest Lyapunov exponents is obtained by computer simulation, which can prove the existence of chaotic phenomena in the system (8). Based on the above theoretical results and numerical experiments, we can know that the development of the system can be effectively controlled by choosing the appropriate impulsive period $T$, the advanced predator release amount $p$, and the reasonable harvest rate $\delta_{i}(i=1,2,3)$. The comprehensive theoretical analysis and numerical simulation can give some guidance to the development of a real ecosystem and the balance of nature. Controlling the number of insect populations is critical because sudden outbreaks of insects can disrupt ecological balance and affect economic growth. Chemical control and biological control are very important methods for controlling agricultural pests. The usual measure for chemical control is spraying pesticides. Chemical control can kill pests quickly and effectively and reduce economic losses, but it also causes certain pollution to the environment. In order to reduce the environmental pollution caused by chemical control, biological control can be 
achieved by stocking or releasing natural enemies, but its effect is not obvious and rapid. Therefore, integrated control is a very effective means of balancing. The results obtained in this work provide a useful platform for pest control, fishery production, species breeding, and other fields.

\section{Data Availability}

Data sharing is not applicable to this article as no data sets were generated or analyzed during the current study.

\section{Conflicts of Interest}

The authors declare that they have no conflicts of interest in the work.

\section{Acknowledgments}

This work was supported by the NNSF of China (Grant no. 61773014), the program of China Scholarships Council (no. 201906840070), NSF of Guangxi (Grant no. 2018GXNSFAA294010), Middle-aged and Young Teachers' Basic Ability Promotion Project Fund of Guangxi Colleges (Grant no. 2019KY0669), SF of Guangxi University of Finance and Economics (Grant no. 2019XTZZ06), and Guangxi Key Laboratory Cultivation Base of Cross-border E-commerce Intelligent Information Processing.

\section{References}

[1] X. Ma, Y. Shao, Z. Wang, M. Luo, X. Fang, and Z. Ju, “An impulsive two-stage predator-prey model with stage-structure and square root functional responses," Mathematics and Computers in Simulation, vol. 119, pp. 91-107, 2016.

[2] Y. Shao and Y. Li, "Dynamical analysis of a stage structured predator-prey system with impulsive diffusion and generic functional response," Applied Mathematics and Computation, vol. 220, pp. 472-481, 2013.

[3] H. Baek, "Dynamic complexities of a three-species beddington-deangelis system with impulsive control strategy," Acta Applicandae Mathematicae, vol. 110, no. 1, pp. 23-38, 2010.

[4] Z. Wang, Y. Shao, X. Fang, and X. Ma, "The dynamic behaviors of one-predator two-prey system with mutual interference and impulsive control," Mathematics and Computers in Simulation, vol. 132, pp. 68-85, 2017.

[5] G. Buffoni, M. Groppi, and C. Soresina, "Effects of prey over-undercrowding in predator-prey systems with preydependent trophic functions," Nonlinear Analysis: Real World Applications, vol. 12, no. 5, pp. 2871-2887, 2011.

[6] L. Galbusera and S. Pasquali, "Analysis and constrained optimal impulsive control of a Holling-II type trophic system with two sources," Journal of the Franklin Institute, vol. 352, no. 7, pp. 2728-2749, 2015.

[7] K. Yang, "Basic properties of mathematical population models," International Journal of Biomathematics, vol. 17, no. 2, pp. 129-142, 2002.

[8] H. Baek, "Species extinction and permanence of an impulsively controlled two-prey one-predator system with seasonal effects," BioSystems, vol. 98, no. 1, pp. 7-18, 2009.

[9] M. Huang, J. Li, X. Song, and H. Guo, "Modeling impulsive injections of insulin: towards artificial pancreas," SIAM
Journal on Applied Mathematics, vol. 72, no. 5, pp. 1524-1548, 2012.

[10] Y. Zhang, B. Liu, and L. Chen, "Extinction and permanence of a two-prey one-predator system with impulsive effect," Mathematical Medicine and Biology, vol. 20, no. 4, pp. 309325, 2003.

[11] B. Sahoo and S. Poria, "Disease control in a food chain model supplying alternative food," Applied Mathematical Modelling, vol. 37, no. 8, pp. 5653-5663, 2013.

[12] B. Sahoo and S. Poria, "Diseased prey predator model with general holling type interactions," Applied Mathematics and Computation, vol. 226, pp. 83-100, 2014.

[13] J. Ghosh, B. Sahoo, and S. Poria, "Prey-predator dynamics with prey refuge providing additional food to predator," Chaos, Solitons \& Fractals, vol. 96, pp. 110-119, 2017.

[14] R. D. Holt, "Predation, apparent competition, and the structure of prey communities," Theoretical Population Biology, vol. 12, no. 2, pp. 197-229, 1977.

[15] R. D. Holt, "On the evolutionary stability of sink populations," Evolutionary Ecology, vol. 11, no. 6, pp. 723-731, 1997.

[16] H. Wang and W. Wang, "The dynamical complexity of a Ivlev-type prey-predator system with impulsive effect," Chaos, Solitons \& Fractals, vol. 38, no. 4, pp. 1168-1176, 2008.

[17] G. Seo and D. L. DeAngelis, "A predator-prey model with a Holling type I functional response including a predator mutual interference," Journal of Nonlinear Science, vol. 21, no. 6, pp. 811-833, 2011.

[18] X. Song and Y. Li, "Dynamic behaviors of the periodic predator-prey model with modified leslie-gower Holling-type II schemes and impulsive effect," Nonlinear Analysis: Real World Applications, vol. 9, no. 1, pp. 64-79, 2008.

[19] Z. Ju, Y. Shao, W. Kong, X. Ma, and X. Fang, “An impulsive prey-predator system with stage-structure and Holling II functional response," Advances in Difference Equations, vol. 2014, no. 1, p. 280, 2014.

[20] K. Yang, Delay Differential Equations: with Applications in Population Dynamics, vol. 191, Academic Press, Cambridge, MA, USA, 1993.

[21] W. G. Aiello and H. I. Freedman, "A time-delay model of single-species growth with stage structure," Mathematical Biosciences, vol. 101, no. 2, pp. 139-153, 1990.

[22] X. Wang and X. Song, "Mathematical models for the control of a pest population by infected pest," Computers \& Mathematics with Applications, vol. 56, no. 1, pp. 266-278, 2008.

[23] H. Guo and L. Chen, "The effects of impulsive harvest on a predator-prey system with distributed time delay," Communications in Nonlinear Science and Numerical Simulation, vol. 14, no. 5, pp. 2301-2309, 2009.

[24] K. Liu, X. Meng, and L. Chen, "A new stage structured predator-prey gomportz model with time delay and impulsive perturbations on the prey," Applied Mathematics and Computation, vol. 196, no. 2, pp. 705-719, 2008.

[25] H. Yu, S. Zhong, and M. Ye, "Dynamic analysis of an ecological model with impulsive control strategy and distributed time delay," Mathematics and Computers in Simulation, vol. 80, no. 3, pp. 619-632, 2009.

[26] M. Zhao, X. Wang, H. Yu, and J. Zhu, "Dynamics of an ecological model with impulsive control strategy and distributed time delay," Mathematics and Computers in Simulation, vol. 82, no. 8, pp. 1432-1444, 2012.

[27] M. P. Hassell, "Density-dependence in single-species populations," The Journal of Animal Ecology, vol. 44, no. 1, pp. 283-295, 1975. 
[28] K. Wang and Y. Zhu, "Global attractivity of positive periodic solution for a volterra model," Applied Mathematics and Computation, vol. 203, no. 2, pp. 493-501, 2008.

[29] H. Guo and X. Chen, "Existence and global attractivity of positive periodic solution for a volterra model with mutual interference and beddington-deangelis functional response," Applied Mathematics and Computation, vol. 217, no. 12, pp. 5830-5837, 2011.

[30] Z. Du and Y. Lv, "Permanence and almost periodic solution of a lotka-volterra model with mutual interference and time delays," Applied Mathematical Modelling, vol. 37, no. 3, pp. 1054-1068, 2013.

[31] K. Wang, "Existence and global asymptotic stability of positive periodic solution for a predator-prey system with mutual interference," Nonlinear Analysis: Real World Applications, vol. 10, no. 5, pp. 2774-2783, 2009.

[32] M. Zhao, H. Yu, and J. Zhu, "Effects of a population floor on the persistence of chaos in a mutual interference hostparasitoid model," Chaos, Solitons \& Fractals, vol. 42, no. 2, pp. 1245-1250, 2009.

[33] W. M. Schaffer and M. Kot, "Do strange attractors govern ecological systems?," BioScience, vol. 35 , no. 6, pp. 342-350, 1985.

[34] B. Sahoo and S. Poria, "The chaos and control of a food chain model supplying additional food to top-predator," Chaos, Solitons \& Fractals, vol. 58, pp. 52-64, 2014.

[35] V. Lakshmikantham, P. S. Simeonov, and D. Bainov, Theory of Impulsive Differential Equations, vol. 6, World Scientific, Singapore, 1989.

[36] D. Bainov and P. Simeonov, "Impulsive differential equations: periodic solutions and applications," in Pitman Monographs and Surveys in Pure and Applied Mathematics, vol. 66, Longman Scientific and Technical, Harlow, UK, 1993.

[37] F. Grond, H. H. Diebner, S. Sahle, A. Mathias, S. Fischer, and O. E. Rossler, "A robust, locally interpretable algorithm for lyapunov exponents," Chaos, Solitons \& Fractals, vol. 16, no. 5, pp. 841-852, 2003.

[38] C. Masoller, A. C. S. Schifino, and L. Romanelli, "Characterization of strange attractors of lorenz model of general circulation of the atmosphere," Chaos, Solitons \& Fractals, vol. 6, pp. 357-366, 1995.

[39] M. T. Rosenstein, J. J. Collins, and C. J. De Luca, “A practical method for calculating largest lyapunov exponents from small data sets," Physica D: Nonlinear Phenomena, vol. 65, no. 1-2, pp. 117-134, 1993. 


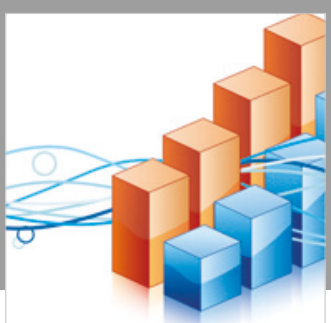

Advances in

Operations Research

\section{-n-m}
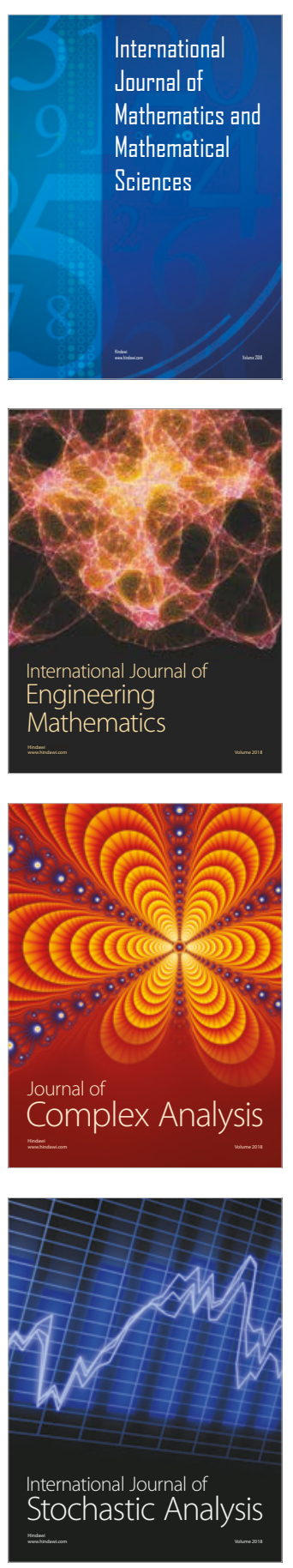
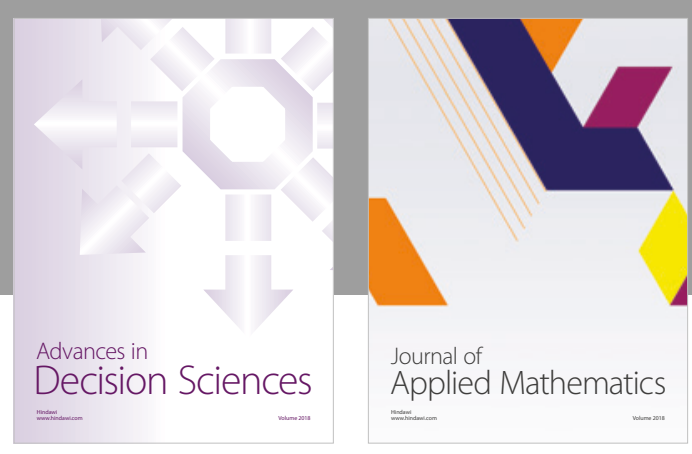

Journal of

Applied Mathematics
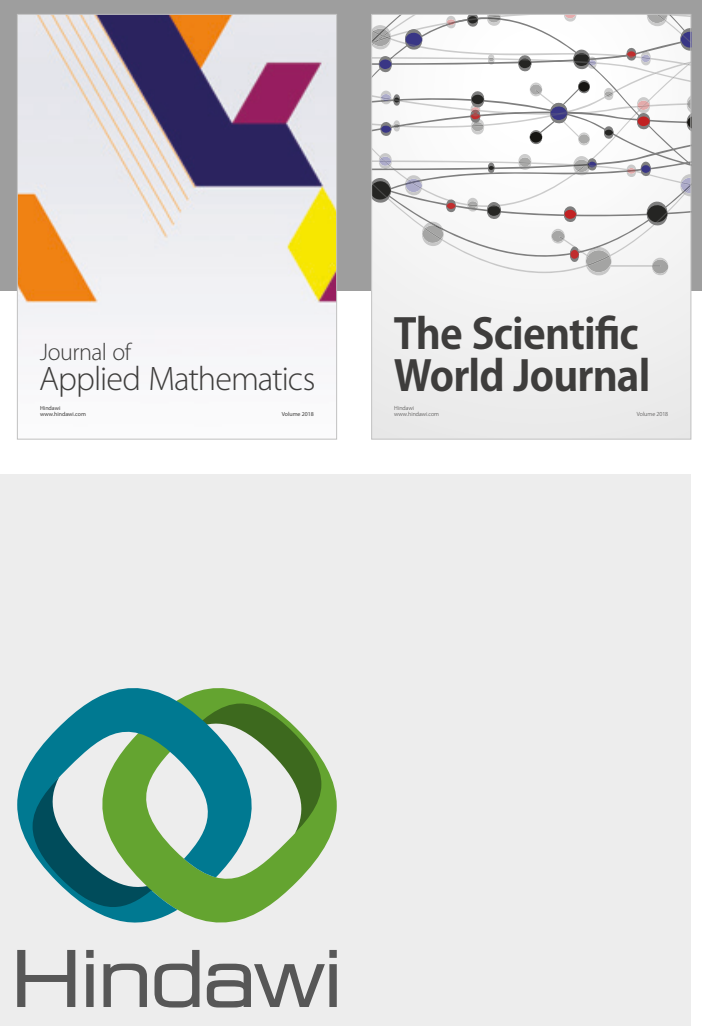

Submit your manuscripts at

www.hindawi.com

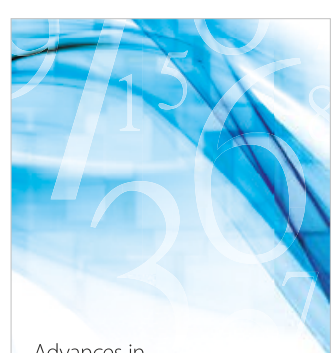

Advances in
Numerical Analysis
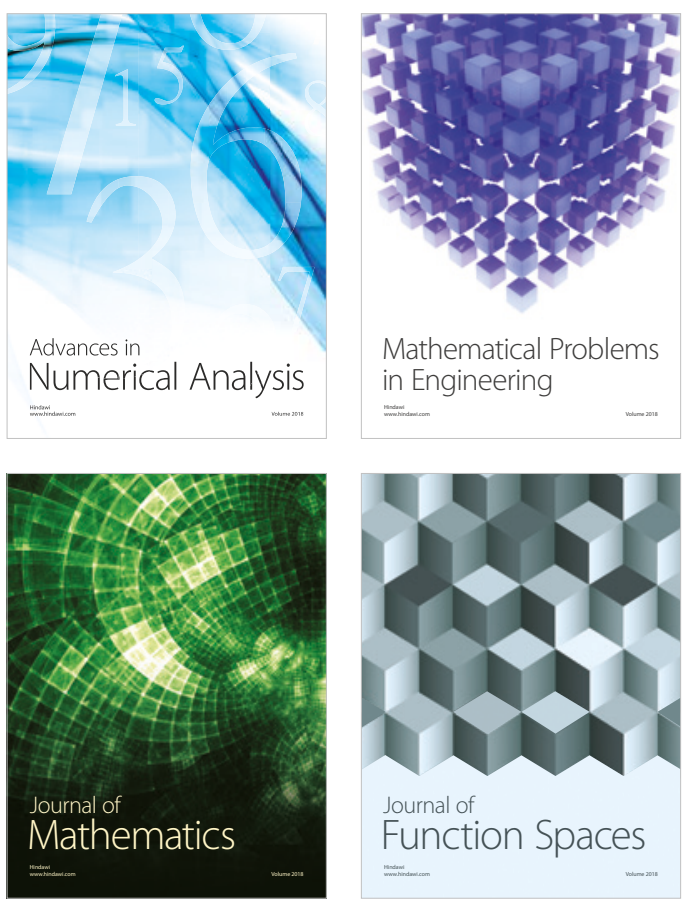

Mathematical Problems in Engineering

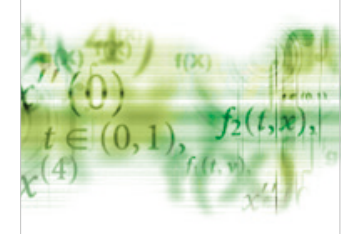

International Journal of

Differential Equations

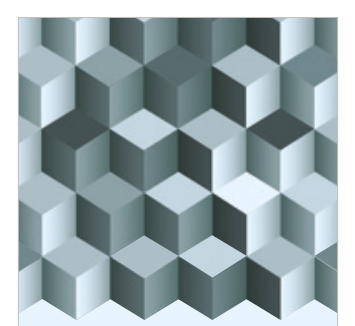

Journal of

Function Spaces

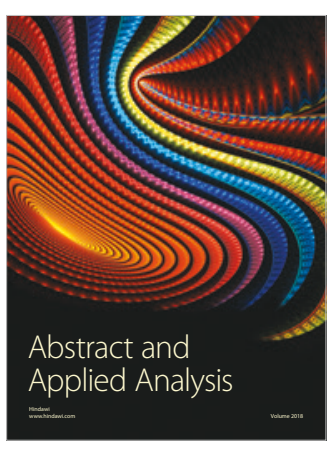

The Scientific

World Journal

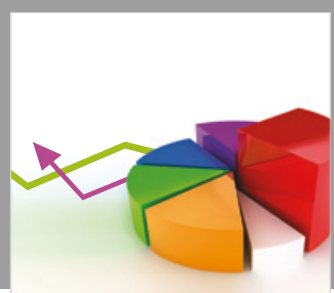

Journal of

Probability and Statistics
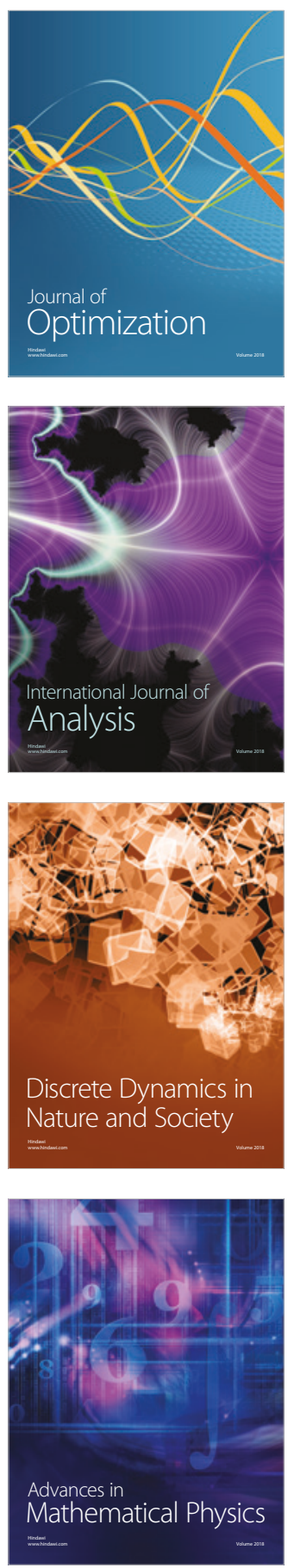